\title{
Direct Oral Anticoagulants: Drug Selection by Means of the SOJA Method
}

\author{
Robert Janknegt $^{1 *}$, Roel Van Kampen ${ }^{2}$, Niels Boone ${ }^{3}$ and Renée Vossen ${ }^{4}$ \\ ${ }^{1}$ Hospital Pharmacist, Clinical Pharmacologist Sittard, The Netherlands \\ ${ }^{2} M D$, PhD, haematologist, Oncologist, Zuyderland Medical Centre, The Netherlands \\ ${ }^{3}$ Hospital Pharmacist, Clinical Pharmacologist, Zuyderland Medical Centre, The Netherlands \\ ${ }^{4}$ Clinical Chemistry Specialist, Zuyderland Medical Centre, The Netherlands \\ *Corresponding Author: Robert Janknegt, Hospital Pharmacist, Clinical \\ Pharmacologist Sittard, The Netherlands.
}

Received: October 30, 2020

Published: November 30, 2020

(c) All rights are reserved by Robert Janknegt., et al.

\begin{abstract}
Objectives: The increasing number of direct acting anticoagulant drugs (DOACs) makes it almost impossible to have sufficient knowledge of each individual medicine and device, especially for general practitioners. Reducing the number of medicines different DOACs, based on rational criteria, allows physicians and pharmacists to build experience with a more limited set of medicines and to optimise patient information.

Methods: In this study DOACs are compared by means of the SOJA method. The following selection criteria were applied: approved indications, available formulations, variability of the AUC, drug interactions, clinical efficacy, side effects, dosage frequency and documentation.

Results: Limited differences in scores were found between apixaban, dabigratran and rivaroxaban. Edoxaban showed a lower score, mostly because of its more limited clinical evidence and documentation. The ranking between the top 3 depends mostly on the assigned weight to the individual selection criteria. Acquisition cost was not taken into account, because this varies with time. In practice acquisition cost is of course an important selection criterion, especially because there are very limited differences between the medicines from a clinical perspective. Exclusion of this criterion also makes this comparison more internationally applicable.

Conclusions: All DOACs are suitable for formulary inclusion, followed by a selection of the most suitable for a DOAC in individual patients, based on patient characteristics.
\end{abstract}

Keywords: Drug; Apixaban; Rivaroxaban; DOAC

Part 2

\begin{tabular}{|c|c|c|c|c|c|c|c|c|}
\hline Medicines & $\begin{array}{c}\text { Duration } \\
\text { Days } \\
\text { (days) }\end{array}$ & $\mathbf{N}$ & $\begin{array}{l}\text { First dose, timing } \\
\text { (hours) }\end{array}$ & Age & $\begin{array}{c}\text { Female } \\
(\%)\end{array}$ & Design & $\begin{array}{c}\text { Duration of } \\
\text { follow-up }\end{array}$ & Ref \\
\hline $\begin{array}{l}\text { Api } 2.5 \mathrm{mg} \text { bid or } \\
\text { Enox } 30 \mathrm{mg} \text { bid sc } \\
\text { War INR } 1.8-3 \text { or }\end{array}$ & $10-14$ & $\begin{array}{l}111 \\
109 \\
109 \\
\end{array}$ & $\begin{array}{c}\text { 12-24 post } \\
12-24 \text { post } \\
\text { Evening of surgery }\end{array}$ & $\begin{array}{l}68 \\
67 \\
67 \\
\end{array}$ & $\begin{array}{l}68 \\
62 \\
61 \\
\end{array}$ & $\begin{array}{c}\text { DB } \\
\text { DB } \\
\text { Open } \\
\end{array}$ & 10-14 days & [26] \\
\hline $\begin{array}{l}\text { Api } 2.5 \mathrm{mg} \text { bid or } \\
\text { Enox } 30 \mathrm{mg} \text { bid sc }\end{array}$ & $10-14$ & $\begin{array}{l}1599 \\
1596 \\
\end{array}$ & $\begin{array}{l}12-24 \text { post } \\
12-24 \text { post }\end{array}$ & $\begin{array}{l}66 \\
66 \\
\end{array}$ & $\begin{array}{l}62 \\
62 \\
\end{array}$ & $\mathrm{DB}, \mathrm{DD}$ & 70 days & [27] \\
\hline $\begin{array}{l}\text { Api } 2.5 \mathrm{mg} \text { bid or } \\
\text { Enox } 40 \mathrm{mg} \text { qd sc }\end{array}$ & $10-14$ & $\begin{array}{l}1528 \\
1529\end{array}$ & $\begin{array}{l}12-24 \text { post } \\
12 \text { before }\end{array}$ & $\begin{array}{l}67 \\
67\end{array}$ & $\begin{array}{l}71 \\
74\end{array}$ & DB, DD & 10-14 days & [28] \\
\hline $\begin{array}{l}\text { Api } 2.5 \mathrm{mg} \text { bid or } \\
\text { Enox } 40 \mathrm{mg} \text { qd sc }\end{array}$ & 35 & $\begin{array}{l}2708 \\
2699 \\
\end{array}$ & $\begin{array}{l}12-24 \text { post } \\
12 \text { before }\end{array}$ & $\begin{array}{l}61 \\
61 \\
\end{array}$ & $\begin{array}{l}53 \\
54 \\
\end{array}$ & $\mathrm{DB}, \mathrm{DD}$ & 95 days & [29] \\
\hline $\begin{array}{l}\text { Dabi } 150 \mathrm{mg} \text { bid } \\
\text { Dabi } 225 \mathrm{mg} \text { bid } \\
\text { Enox } 40 \mathrm{mg} \text { qd }\end{array}$ & $6-10$ & $\begin{array}{l}390 \\
393 \\
392 \\
\end{array}$ & $\begin{array}{l}1-4 \text { post } \\
12 \text { before }\end{array}$ & $\begin{array}{l}66 \\
66 \\
65 \\
\end{array}$ & $\begin{array}{l}65 \\
58 \\
62 \\
\end{array}$ & $\mathrm{DB}, \mathrm{DD}$ & $6-10$ & [30] \\
\hline $\begin{array}{l}\text { Dabi } 150 \mathrm{mg} \text { qd } \\
\text { Dabi } 220 \mathrm{mg} \text { qd } \\
\text { Enox } 40 \mathrm{mg} \text { qd }\end{array}$ & $6-10$ & $\begin{array}{l}703 \\
679 \\
694\end{array}$ & $\begin{array}{l}\text { 1-4 post } \\
\text { evening before }\end{array}$ & $\begin{array}{l}68 \\
67 \\
68 \\
\end{array}$ & $\begin{array}{l}64 \\
65 \\
69 \\
\end{array}$ & $\mathrm{DB}, \mathrm{DD}$ & 3 months & {$[31$} \\
\hline
\end{tabular}




\begin{tabular}{|c|c|c|c|c|c|c|c|c|}
\hline $\begin{array}{l}\text { Dabi } 150 \text { mg qd } \\
\text { Dabi } 220 \text { mg qd } \\
\text { Enox } 40 \mathrm{mg} \mathrm{qd}\end{array}$ & $28-35$ & $\begin{array}{l}1163 \\
1146 \\
1154\end{array}$ & $\begin{array}{l}\text { 1-4 post } \\
\text { evening before }\end{array}$ & $\begin{array}{l}63 \\
65 \\
64\end{array}$ & $\begin{array}{l}57 \\
56 \\
56\end{array}$ & DB, DD & $28-35$ & [32] \\
\hline $\begin{array}{l}\text { Dabi } 220 \text { mg qd } \\
\text { Enox } 40 \mathrm{mg} \mathrm{qd}\end{array}$ & $28-35$ & $\begin{array}{l}1036 \\
1019 \\
\end{array}$ & $\begin{array}{l}1-4 \text { post } \\
\text { evening before }\end{array}$ & $\begin{array}{l}62 \\
62\end{array}$ & $\begin{array}{l}54 \\
50\end{array}$ & $\mathrm{DB}, \mathrm{DD}$ & 90 & [33] \\
\hline $\begin{array}{l}\text { Dabi } 150 \mathrm{mg} \mathrm{qd} \\
\text { Dabi } 220 \mathrm{mg} q \mathrm{~d} \\
\text { Enox } 30 \mathrm{mg} \text { bid }\end{array}$ & $12-15$ & $\begin{array}{l}877 \\
862 \\
876\end{array}$ & $\begin{array}{l}12-24 \text { post } \\
12-24 \text { post }\end{array}$ & $\begin{array}{l}66 \\
66 \\
66\end{array}$ & $\begin{array}{l}58 \\
57 \\
58\end{array}$ & DB, DD & 90 & [34] \\
\hline $\begin{array}{l}\text { Edo } 15 \mathrm{mg} \text { qd } \\
\text { Edo } 30 \mathrm{mg} \text { qd } \\
\text { Dalte } 5000 \mathrm{IU} \text { qd }\end{array}$ & $7-10$ & $\begin{array}{l}170 \\
151 \\
144 \\
\end{array}$ & $\begin{array}{c}\text { 6-8 post } \\
6-8 \text { post } \\
6-8 \\
\end{array}$ & $\begin{array}{l}57 \\
57 \\
58 \\
\end{array}$ & $\begin{array}{l}58 \\
65 \\
61 \\
\end{array}$ & DB, DD & $7-10$ & [35] \\
\hline $\begin{array}{l}\text { Edo } 30 \mathrm{mgqd} \\
\text { Enox } 20 \mathrm{mg} \text { bid }\end{array}$ & $11-14$ & $\begin{array}{l}299 \\
295 \\
\end{array}$ & $\begin{array}{l}\text { 6-24 post } \\
24-36 \text { post } \\
\end{array}$ & $\begin{array}{l}73 \\
72 \\
\end{array}$ & $\begin{array}{l}82 \\
78 \\
\end{array}$ & DB, DD & $11-14$ & [36] \\
\hline $\begin{array}{l}\text { Edo } 15 \mathrm{mg} \text { qd } \\
\text { Edo } 30 \mathrm{mg} \text { qd } \\
\text { Enox } 20 \mathrm{mg} \text { bid } \\
\end{array}$ & $11-14$ & $\begin{array}{l}78 \\
72 \\
74 \\
\end{array}$ & $\begin{array}{l}\text { 6-24 post } \\
24-36 \text { post }\end{array}$ & $\begin{array}{l}61 \\
61 \\
59 \\
\end{array}$ & $\begin{array}{l}81 \\
96 \\
80 \\
\end{array}$ & DB, DD & $11-14$ & [37] \\
\hline $\begin{array}{l}\text { Edo } 30 \mathrm{mg} \text { qd } \\
\text { Enox } 20 \mathrm{mg} \text { bid }\end{array}$ & $11-14$ & $\begin{array}{l}255 \\
248 \\
\end{array}$ & $\begin{array}{l}\text { 6-24 post } \\
24-36 \text { post }\end{array}$ & $\begin{array}{l}63 \\
63 \\
\end{array}$ & $\begin{array}{l}87 \\
86\end{array}$ & DB, DD & $11-14$ & [38] \\
\hline $\begin{array}{l}\text { Riva } 10 \mathrm{mg} \mathrm{qd} \\
\text { Enox } 40 \mathrm{mg} \mathrm{qd}\end{array}$ & 36 & $\begin{array}{l}2266 \\
2275 \\
\end{array}$ & $\begin{array}{l}\text { 6-8 post } \\
\text { Evening before }\end{array}$ & $\begin{array}{l}63 \\
63 \\
\end{array}$ & $\begin{array}{l}55 \\
56\end{array}$ & DB, DD & 70 & [39] \\
\hline $\begin{array}{l}\text { Riva } 10 \mathrm{mg} q \mathrm{q} \\
\text { Enox } 40 \mathrm{mg} q \mathrm{~d}\end{array}$ & $\begin{array}{c}36 \\
10-14 \\
\end{array}$ & $\begin{array}{l}1252 \\
1257 \\
\end{array}$ & $\begin{array}{c}\text { 6-8 post } \\
\text { Evening before }\end{array}$ & $\begin{array}{l}61 \\
62 \\
\end{array}$ & $\begin{array}{l}54 \\
53 \\
\end{array}$ & DB, DD & 70 & [40] \\
\hline $\begin{array}{l}\text { Riva } 10 \mathrm{mg} \mathrm{qd} \\
\text { Enox } 40 \mathrm{mg} \mathrm{qd}\end{array}$ & $10-14$ & $\begin{array}{l}1254 \\
1277 \\
\end{array}$ & $\begin{array}{l}6-8 \text { post } \\
12 \text { before }\end{array}$ & $\begin{array}{l}68 \\
68 \\
\end{array}$ & $\begin{array}{l}70 \\
66 \\
\end{array}$ & DB, DD & 50 & [41] \\
\hline $\begin{array}{l}\text { Riva } 10 \mathrm{mg} \text { qd } \\
\text { Enox } 30 \mathrm{mg} \text { bid }\end{array}$ & $10-14$ & $\begin{array}{l}1254 \\
1277 \\
\end{array}$ & $\begin{array}{l}6-8 \text { post } \\
12 \text { post }\end{array}$ & $\begin{array}{l}64 \\
65\end{array}$ & $\begin{array}{l}66 \\
64 \\
\end{array}$ & DB, DD & 50 & [42] \\
\hline $\begin{array}{l}\text { Riva } 10 \mathrm{mg} q \mathrm{~d} \\
\text { Aspirin } 81 \mathrm{mg} \text { qd }\end{array}$ & 9 or 30 & $\begin{array}{l}1717 \\
1707\end{array}$ & $\begin{array}{c}\text { After } 5 \text { days rivaroxaban open } \\
\text { label }\end{array}$ & $\begin{array}{l}63 \\
63\end{array}$ & $\begin{array}{l}51 \\
53\end{array}$ & DB, DD & 90 & [43] \\
\hline
\end{tabular}

Table 1: Comparative studies in orthopaedic surgery, baseline data and design.

\begin{tabular}{|c|c|c|c|c|c|c|}
\hline Medicines & BMI & $\begin{array}{c}\text { Arthritis } \\
\text { (\%) }\end{array}$ & $\begin{array}{l}\text { Duration of } \\
\text { Surgery (h) }\end{array}$ & Surgery & Primary endpoint & Ref \\
\hline Api $2.5 \mathrm{mg}$ bid or & 31 & 70 & 1.4 & \multirow[t]{3}{*}{ Knee } & \multirow{3}{*}{$\begin{array}{c}\text { VTE-A + VTE-S + } \\
\text { PE-NF }\end{array}$} & \multirow[t]{3}{*}[26]{} \\
\hline Enox $30 \mathrm{mg}$ bid sc & 30 & 71 & 1.6 & & & \\
\hline War INR $1.8-3$ or & 30 & 72 & 1.6 & & & \\
\hline Api $2.5 \mathrm{mg}$ bid or & 31 & 81 & 1.5 & \multirow[t]{2}{*}{ Knee } & \multirow{2}{*}{$\begin{array}{c}\text { VTE-A + VTE-S + } \\
\text { PE-NF+ death }\end{array}$} & \multirow[t]{2}{*}[27]{} \\
\hline Enox $30 \mathrm{mg}$ bid sc & 31 & 80 & 1.5 & & & \\
\hline Api $2.5 \mathrm{mg}$ bid or & 29 & 63 & 1.6 & \multirow[t]{2}{*}{ Knee } & \multirow{2}{*}{$\begin{array}{c}\mathrm{VTE}+\mathrm{PE}-\mathrm{NF}+ \\
\text { death }\end{array}$} & \multirow[t]{2}{*}[28]{} \\
\hline Enox 40 mg qd sc & 29 & 63 & 1.6 & & & \\
\hline Api $2.5 \mathrm{mg}$ bid or & 28 & 57 & 1.5 & \multirow[t]{2}{*}{ Hip } & \multirow{2}{*}{$\begin{array}{c}\text { VTE + PE-NF+ } \\
\text { death }\end{array}$} & \multirow[t]{2}{*}[29]{} \\
\hline Enox 40 mg qd sc & 28 & 58 & 1.5 & & & \\
\hline
\end{tabular}




\begin{tabular}{|c|c|c|c|c|c|c|}
\hline $\begin{array}{l}\text { Dabi } 150 \mathrm{mg} \text { bid } \\
\text { Dabi } 225 \mathrm{mg} \text { bid } \\
\text { Enox } 40 \mathrm{mg} \mathrm{qd}\end{array}$ & & & $\begin{array}{l}1.5 \\
1.4 \\
1.5\end{array}$ & Hip and knee & $\begin{array}{l}\text { VTE during treat- } \\
\text { ment }\end{array}$ & [30] \\
\hline $\begin{array}{l}\text { Dabi } 150 \text { mg qd } \\
\text { Dabi } 220 \mathrm{mg} \text { qd } \\
\text { Enox } 40 \mathrm{mg} \mathrm{qd}\end{array}$ & & & $\begin{array}{l}1.5 \\
1.5 \\
1.5\end{array}$ & Knee & VTE + mortality & [31] \\
\hline $\begin{array}{l}\text { Dabi } 150 \mathrm{mg} \mathrm{qd} \\
\text { Dabi } 220 \mathrm{mg} \mathrm{qd} \\
\text { Enox } 40 \mathrm{mg} \mathrm{qd}\end{array}$ & & & $\begin{array}{l}1.5 \\
1.5 \\
1.5 \\
\end{array}$ & Hip & VTE + mortality & [32] \\
\hline $\begin{array}{l}\text { Dabi } 220 \mathrm{mg} \mathrm{qd} \\
\text { Enox } 40 \mathrm{mg} \mathrm{qd}\end{array}$ & $\begin{array}{l}28 \\
28 \\
\end{array}$ & & $\begin{array}{l}1.3 \\
1.3 \\
\end{array}$ & Hip & VTE + mortality & [33] \\
\hline $\begin{array}{l}\text { Dabi } 150 \mathrm{mg} \mathrm{qd} \\
\text { Dabi } 220 \mathrm{mg} \text { qd } \\
\text { Enox } 30 \mathrm{mg} \text { bid }\end{array}$ & & & $\begin{array}{l}1.5 \\
1.5 \\
1.5 \\
\end{array}$ & Knee & VTE + mortality & [34] \\
\hline $\begin{array}{l}\text { Edo } 15 \mathrm{mg} \text { qd } \\
\text { Edo } 30 \mathrm{mg} \text { qd } \\
\text { Dalte } 5000 \mathrm{IU} \text { qd }\end{array}$ & $\begin{array}{l}27 \\
28 \\
28 \\
\end{array}$ & & $\begin{array}{l}1.4 \\
1.4 \\
1.4 \\
\end{array}$ & Knee & VTE & [35] \\
\hline $\begin{array}{l}\text { Edo } 30 \mathrm{mg} \mathrm{qd} \\
\text { Enox } 20 \mathrm{mg} \text { bid }\end{array}$ & & $\begin{array}{l}87 \\
87 \\
\end{array}$ & $\begin{array}{l}1.5 \\
1.5 \\
\end{array}$ & Knee & VTE & [36] \\
\hline $\begin{array}{l}\text { Edo } 15 \mathrm{mg} \text { qd } \\
\text { Edo } 30 \mathrm{mg} \text { qd } \\
\text { Enox } 20 \mathrm{mg} \text { bid }\end{array}$ & $\begin{array}{l}24 \\
24 \\
23 \\
\end{array}$ & & $\begin{array}{l}1.5 \\
1.5 \\
1.6 \\
\end{array}$ & Knee & VTE & [37] \\
\hline $\begin{array}{l}\text { Edo } 30 \mathrm{mg} \text { qd } \\
\text { Enox } 20 \mathrm{mg} \text { bid }\end{array}$ & $\begin{array}{l}25 \\
24 \\
\end{array}$ & & & Hip & VTE & [38] \\
\hline $\begin{array}{l}\text { Riva } 10 \mathrm{mg} \mathrm{qd} \\
\text { Enox } 40 \mathrm{mg} \text { qd }\end{array}$ & $\begin{array}{l}28 \\
28 \\
\end{array}$ & & $\begin{array}{l}1.5 \\
1.5 \\
\end{array}$ & Hip & $\begin{array}{l}\text { DVT + PE-NF + } \\
\text { death from VTE }\end{array}$ & [39] \\
\hline $\begin{array}{l}\text { Riva } 10 \mathrm{mg} \mathrm{qd} \\
\text { Enox } 40 \mathrm{mg} \text { qd }\end{array}$ & $\begin{array}{l}27 \\
27 \\
\end{array}$ & & $\begin{array}{l}1.6 \\
1.6 \\
\end{array}$ & Hip & $\begin{array}{c}\text { DVT + PE-NF + } \\
\text { death }\end{array}$ & {$[40]$} \\
\hline $\begin{array}{l}\text { Riva } 10 \mathrm{mg} q \mathrm{~d} \\
\text { Enox } 40 \mathrm{mg} \text { qd }\end{array}$ & $\begin{array}{l}30 \\
30 \\
\end{array}$ & & $\begin{array}{l}1.6 \\
1.6 \\
\end{array}$ & Knee & $\begin{array}{c}\text { DVT + PE-NF + } \\
\text { death }\end{array}$ & {$[41]$} \\
\hline $\begin{array}{l}\text { Riva } 10 \mathrm{mg} \text { qd } \\
\text { Enox } 30 \mathrm{mg} \text { bid }\end{array}$ & $\begin{array}{l}31 \\
31 \\
\end{array}$ & & $\begin{array}{l}1.6 \\
1.6 \\
\end{array}$ & Knee & $\begin{array}{c}\text { DVT + PE-NF + } \\
\text { death }\end{array}$ & {$[42]$} \\
\hline $\begin{array}{l}\text { Riva } 10 \mathrm{mg} \text { qd } \\
\text { Aspirin } 81 \mathrm{mg} \text { qd }\end{array}$ & $\begin{array}{l}31 \\
31\end{array}$ & & $\begin{array}{l}1.4 \\
1.4\end{array}$ & Knee and hip & DVT-S & {$[43]$} \\
\hline
\end{tabular}

Table 2: Comparative studies in orthopaedic surgery, baseline data and design. 


\begin{tabular}{|c|c|c|c|c|c|c|c|c|c|c|c|c|}
\hline Medicines & $\begin{array}{c}\text { DVT-A } \\
\text { (\%) }\end{array}$ & $\begin{array}{c}\text { DVT-S } \\
\text { (\%) }\end{array}$ & $\begin{array}{c}\text { DVT-M } \\
\text { (\%) }\end{array}$ & $\begin{array}{c}\text { DVT-P } \\
\text { (\%) }\end{array}$ & $\begin{array}{l}\text { DVT } \\
\text { (\%) }\end{array}$ & $\begin{array}{l}\text { VTE } \\
\text { (\%) }\end{array}$ & $\begin{array}{l}\text { PE } \\
\text { (\%) }\end{array}$ & $\begin{array}{c}\text { Fatal } \\
\text { PE } \\
(\%) \\
\end{array}$ & $\begin{array}{l}\text { Mor- } \\
\text { tality } \\
\text { (\%) }\end{array}$ & $\begin{array}{c}\text { Composite } \\
\text { Efficacy } \\
(\%) \\
\end{array}$ & & $\begin{array}{c}\text { Ref- } \\
\text { er- } \\
\text { ence }\end{array}$ \\
\hline $\begin{array}{l}\text { Api } 2.5 \mathrm{mg} \text { bid } \\
\text { Enox } 30 \mathrm{mg} \text { bid } \\
\text { War INR } 1.8-3\end{array}$ & $\begin{array}{c}8.3 \\
12.8 \\
25.7\end{array}$ & $\begin{array}{l}0.9 \\
0.9 \\
0.9\end{array}$ & & $\begin{array}{l}0.9 \\
2.8 \\
1.8\end{array}$ & & $\begin{array}{l}10 \\
17 \\
29\end{array}$ & $\begin{array}{c}0 \\
1.8 \\
0\end{array}$ & & $\begin{array}{c}0.7 \\
0 \\
0\end{array}$ & $\begin{array}{c}1.8 \\
4.6 \\
1.8 \\
\text { (VTE-P + PE + death) }\end{array}$ & & [26] \\
\hline $\begin{array}{l}\text { Api } 2.5 \mathrm{mg} \text { bid } \\
\text { Enox } 30 \mathrm{mg} \text { bid } \\
\text { (all results after } \\
10-14 \text { days) }\end{array}$ & & $\begin{array}{l}0.2 \\
0.4\end{array}$ & & $\begin{array}{l}0.7 \\
0.9\end{array}$ & $\begin{array}{l}7.8 \\
8.2\end{array}$ & & $\begin{array}{l}1.0 \\
0.4\end{array}$ & $\begin{array}{c}0.1 \\
0\end{array}$ & $\begin{array}{l}0.2 \\
0.2\end{array}$ & $\begin{array}{c}9.0 \\
8.8 \\
(\mathrm{VTE}+\mathrm{PE}+\text { death })\end{array}$ & $\begin{array}{c}2.0 \\
1.6 \\
\begin{array}{c}\text { (Major VTE + } \\
\text { death }\end{array} \\
\end{array}$ & [27] \\
\hline $\begin{array}{l}\text { Api } 2.5 \mathrm{mg} \text { bid } \\
\text { Enox } 40 \mathrm{mg} \mathrm{qd}\end{array}$ & & $\begin{array}{l}0.2 \\
0.5\end{array}$ & $\begin{array}{c}1.1 \\
2.1 \\
P=0.019\end{array}$ & $\begin{array}{l}0.8 \\
2.2\end{array}$ & $\begin{array}{l}14.6 \\
24.4\end{array}$ & & $\begin{array}{c}0.3 \\
0\end{array}$ & $\begin{array}{c}0.1 \\
0\end{array}$ & $\begin{array}{c}0.13 \\
0\end{array}$ & $\begin{array}{c}15.1 \\
24.4 \\
(\mathrm{VTE}+\mathrm{PE}+\text { death }) \\
\mathrm{P}<0.0001\end{array}$ & & [28] \\
\hline $\begin{array}{l}\text { Api } 2.5 \mathrm{mg} \text { bid } \\
\text { Enox } 40 \mathrm{mg} \text { qd } \\
\text { (all results after } \\
35 \text { days) }\end{array}$ & & $\begin{array}{l}<0.1 \\
0.2\end{array}$ & $\begin{array}{c}0.5 \\
1.1 \\
P=0.01\end{array}$ & $\begin{array}{l}0.3 \\
0.9\end{array}$ & $\begin{array}{l}1.1 \\
3.6\end{array}$ & & $\begin{array}{c}<0.1 \\
0.2\end{array}$ & $\begin{array}{c}<0.1 \\
0\end{array}$ & $\begin{array}{l}0.1 \\
<0.1\end{array}$ & $\begin{array}{c}1.4 \\
3.9 \\
(\mathrm{VTE}+\mathrm{PE}+\text { death }) \\
\mathrm{P}<0.001\end{array}$ & & [29] \\
\hline $\begin{array}{l}\text { Dabi } 150 \mathrm{mg} \text { bid } \\
\text { Dabi } 225 \mathrm{mg} \text { bid } \\
\text { Enox } 40 \mathrm{mg} \text { qd }\end{array}$ & & & & $\begin{array}{l}3.3 \\
1.7 \\
5.6\end{array}$ & $\begin{array}{l}16.8 \\
13.1 \\
24.0\end{array}$ & $\begin{array}{l}17.4 \\
13.1 \\
24.0\end{array}$ & & & & $\begin{array}{c}4.0 \\
1.7 \\
5.6 \\
\text { (DVT/PE) }\end{array}$ & & [30] \\
\hline $\begin{array}{l}\text { Dabi } 150 \mathrm{mg} \text { qd } \\
\text { Dabi } 225 \mathrm{mg} \text { qd } \\
\text { Enox } 40 \mathrm{mg} \text { qd }\end{array}$ & $\begin{array}{l}39.7 \\
36.0 \\
36.0\end{array}$ & $\begin{array}{l}0.4 \\
0.1 \\
1.2\end{array}$ & & $\begin{array}{l}3.4 \\
2.6 \\
3.1\end{array}$ & & & $\begin{array}{c}0.1 \\
0 \\
0.1\end{array}$ & & $\begin{array}{l}0.1 \\
0.1 \\
0.1\end{array}$ & $\begin{array}{l}40.5 \\
36.4 \\
37.7\end{array}$ & & [31] \\
\hline $\begin{array}{l}\text { Dabi } 150 \mathrm{mg} \text { qd } \\
\text { Dabi } 220 \mathrm{mg} \text { qd } \\
\text { Enox } 40 \mathrm{mg} \text { qd }\end{array}$ & $\begin{array}{l}7.2 \\
4.6 \\
6.3\end{array}$ & $\begin{array}{l}0.8 \\
0.5 \\
0.1\end{array}$ & & $\begin{array}{l}3.2 \\
2.0 \\
3.5\end{array}$ & & & $\begin{array}{l}0.1 \\
0.4 \\
0.3\end{array}$ & & $\begin{array}{c}0.3 \\
0.3 \\
0\end{array}$ & $\begin{array}{c}8.6 \\
6.0 \\
6.7 \\
\text { VTE + death }\end{array}$ & $\begin{array}{c}4.3 \\
3.1 \\
3.9 \\
\begin{array}{c}\text { VTE-M + VTE } \\
\text { mortality }\end{array}\end{array}$ & [32] \\
\hline $\begin{array}{l}\text { Dabi } 220 \text { mg qd } \\
\text { Enox } 40 \text { mg qd } \\
\text { (all results after } \\
35 \text { days) }\end{array}$ & & $\begin{array}{c}0 \\
0.4\end{array}$ & & $\begin{array}{c}2.1 \\
3.9 \\
\mathrm{P}=0.04\end{array}$ & $\begin{array}{l}7.6 \\
8.6\end{array}$ & & $\begin{array}{l}0.1 \\
0.2\end{array}$ & & $\begin{array}{c}0 \\
0.1\end{array}$ & $\begin{array}{c}7.7 \\
8.7 \\
\text { VTE + death }\end{array}$ & $\begin{array}{c}2.2 \\
4.2 \\
\begin{array}{c}\text { VTE-M + VTE } \\
\text { mortality }\end{array}\end{array}$ & [33] \\
\hline
\end{tabular}




\begin{tabular}{|c|c|c|c|c|c|c|c|c|c|c|c|}
\hline $\begin{array}{l}\text { Dabi } 150 \mathrm{mg} \text { qd } \\
\text { Dabi } 220 \mathrm{mg} \text { qd } \\
\text { Enox } 40 \mathrm{mg} \text { qd } \\
\text { (all results after } \\
12-15 \text { days) }\end{array}$ & & & & $\begin{array}{l}3.1 \\
2.3 \\
1.6\end{array}$ & & & $\begin{array}{c}0 \\
1.0 \\
0.8\end{array}$ & & $\begin{array}{c}0.2 \\
0.2 \\
0\end{array}$ & $\begin{array}{c}33.7 \\
31.1 \\
25.3 \\
\text { VTE + death } \\
\mathrm{P}=0.02 \\
\mathrm{P}<0.001\end{array}$ & [34] \\
\hline $\begin{array}{l}\text { Edo } 15 \mathrm{mg} \mathrm{qd} \\
\text { Edo } 30 \mathrm{mg} \text { qd } \\
\text { Dalte } 5000 \mathrm{IU} \text { qd }\end{array}$ & & & $\begin{array}{c}6.5 \\
3.3 \\
13.9 \\
\text { (VTE-M) } \\
P=0.036 \\
P<0.001\end{array}$ & $\begin{array}{c}6.5 \\
3.3 \\
13.9 \\
P=0.036 \\
P<0.001\end{array}$ & & $\begin{array}{c}28.2 \\
21.2 \\
43.8 \\
\text { VTE } \\
P=0.005 \\
P<0.001\end{array}$ & & & & & [35] \\
\hline $\begin{array}{l}\text { Edo } 30 \mathrm{mg} \text { qd } \\
\text { Enox } 20 \mathrm{mg} \text { bid }\end{array}$ & $\begin{array}{r}6.0 \\
13.6 \\
\end{array}$ & $\begin{array}{l}1.3 \\
0.3 \\
\end{array}$ & & $\begin{array}{c}0 \\
0.3 \\
\end{array}$ & & $\begin{array}{r}7.4 \\
13.9 \\
\end{array}$ & $\begin{array}{l}0 \\
0 \\
\end{array}$ & $\begin{array}{l}0 \\
0 \\
\end{array}$ & & & [36] \\
\hline $\begin{array}{l}\text { Edo } 15 \mathrm{mg} \text { qd } \\
\text { Edo } 30 \mathrm{mg} \text { qd } \\
\text { Enox } 20 \mathrm{mg} \text { bid }\end{array}$ & & $\begin{array}{l}0 \\
0 \\
0\end{array}$ & & $\begin{array}{l}0 \\
0 \\
0\end{array}$ & $\begin{array}{l}3.8 \\
2.8 \\
4.1\end{array}$ & $\begin{array}{l}3.8 \\
2.8 \\
4.1\end{array}$ & $\begin{array}{l}0 \\
0 \\
0\end{array}$ & $\begin{array}{l}0 \\
0 \\
0\end{array}$ & $\begin{array}{l}0 \\
0 \\
0\end{array}$ & & [37] \\
\hline $\begin{array}{l}\text { Edo } 30 \mathrm{mg} \text { qd } \\
\text { Enox } 20 \mathrm{mg} \text { bid }\end{array}$ & $\begin{array}{l}2.4 \\
6.9\end{array}$ & $\begin{array}{l}0 \\
0\end{array}$ & & $\begin{array}{l}0.4 \\
0.8\end{array}$ & $\begin{array}{l}2.4 \\
6.9\end{array}$ & & $\begin{array}{l}0 \\
0\end{array}$ & $\begin{array}{l}0 \\
0\end{array}$ & $\begin{array}{l}0 \\
0\end{array}$ & $\begin{array}{c}0.4 \\
0.8 \\
\text { DVT-S + DVT-P + PE + } \\
\text { VTE related death }\end{array}$ & [38] \\
\hline $\begin{array}{l}\text { Riva } 10 \mathrm{mg} \text { qd } \\
\text { Enox } 40 \mathrm{mg} \text { qd } \\
\text { (all results after } \\
36 \text { days) }\end{array}$ & & $\begin{array}{l}0.3 \\
0.5\end{array}$ & $\begin{array}{c}0.2 \\
2.0 \\
P<0.001\end{array}$ & $\begin{array}{c}0.1 \\
2.0 \\
P<0.001\end{array}$ & $\begin{array}{c}0.8 \\
3.4 \\
P<0.001\end{array}$ & & $\begin{array}{l}0.3 \\
0.1\end{array}$ & & $\begin{array}{l}0.3 \\
0.3\end{array}$ & 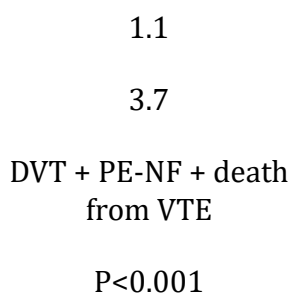 & [39] \\
\hline $\begin{array}{l}\text { Riva } 10 \mathrm{mg} \text { qd } \\
\text { Enox } 40 \mathrm{mg} \text { qd } \\
\text { (all results after } \\
36 \text { days) }\end{array}$ & & $\begin{array}{c}0.2 \\
1.2 \\
P=0.004\end{array}$ & $\begin{array}{c}0.6 \\
5.1 \\
P<0.0001\end{array}$ & $\begin{array}{c}0.6 \\
5.1 \\
P<0.0001\end{array}$ & $\begin{array}{c}1.6 \\
8.2 \\
P<0.0001\end{array}$ & & $\begin{array}{l}0.1 \\
0.5\end{array}$ & & $\begin{array}{l}0.2 \\
0.7\end{array}$ & $\begin{array}{c}2.0 \\
9.3 \\
\text { DVT + PE-NF + death } \\
\mathrm{P}<0.0001\end{array}$ & {$[40]$} \\
\hline
\end{tabular}




\begin{tabular}{|c|c|c|c|c|c|c|c|c|c|c|}
\hline $\begin{array}{l}\text { Riva } 10 \mathrm{mg} \text { qd } \\
\text { Enox } 30 \mathrm{mg} \text { bid } \\
\text { (all results after } \\
15 \text { days) }\end{array}$ & & & $\begin{array}{c}1.0 \\
2.6 \\
P=0.01\end{array}$ & $\begin{array}{l}1.1 \\
2.3\end{array}$ & $\begin{array}{c}9.6 \\
18.2 \\
P<0.001\end{array}$ & $\begin{array}{c}0 \\
0.5\end{array}$ & & $\begin{array}{c}0 \\
0.2\end{array}$ & $\begin{array}{c}9.6 \\
18.9 \\
\text { DVT + PE-NF + death } \\
\mathrm{P}<0.001\end{array}$ & [41] \\
\hline $\begin{array}{l}\text { Riva } 10 \mathrm{mg} \text { qd } \\
\text { Enox } 40 \mathrm{mg} \text { qd } \\
\text { (all results after } \\
15 \text { days) }\end{array}$ & $\begin{array}{l}3.3 \\
5.0\end{array}$ & $\begin{array}{l}0.7 \\
1.2\end{array}$ & $\begin{array}{l}1.1 \\
1.5\end{array}$ & $\begin{array}{l}0.2 \\
0.9\end{array}$ & & $\begin{array}{l}0.3 \\
0.5\end{array}$ & 0 & $\begin{array}{l}0.1 \\
0.2\end{array}$ & $\begin{array}{c}6.7 \\
9.3 \\
\text { DVT + PE-NF + death } \\
P=0.036\end{array}$ & [42] \\
\hline $\begin{array}{l}\text { Riva } 10 \mathrm{mg} \text { qd } \\
\text { Aspirin } 81 \mathrm{mg} \\
\text { qd }\end{array}$ & & $\begin{array}{l}0.70 \\
0.64\end{array}$ & & $\begin{array}{l}0.23 \\
0.23\end{array}$ & & $\begin{array}{l}0.35 \\
0.29\end{array}$ & & $\begin{array}{c}0 \\
0.1\end{array}$ & & [43] \\
\hline
\end{tabular}

Table 3: Comparative studies in orthopaedic surgery, results.

DVT-A: Asymptomatic deep vein thrombosis

DVT-S: Severe/symptomatic deep vein thrombosis

DVT-M: Major deep vein thrombosis

DVT-P: Proximal deep vein thrombosis

PE: Pulmonary embolism

PE-NF: Non-fatal pulmonary embolism

PE-F: Fatal pulmonary embolism

VTE: Venous thromboembolism (ep vein thrombosis plus pulmonary embolism).

\begin{tabular}{|c|c|c|c|c|c|c|c|c|c|c|}
\hline Medicines & $\begin{array}{c}\text { With- } \\
\text { drawal } \\
\text { AE } \\
(\%)\end{array}$ & AE (\%) & $\begin{array}{l}\text { AE drug } \\
\text { related } \\
(\%)\end{array}$ & $\begin{array}{l}\text { AE-S } \\
(\%)\end{array}$ & $\begin{array}{c}\text { Wound } \\
\text { related } \\
\text { infections } \\
(\%)\end{array}$ & $\begin{array}{l}\text { MI } \\
\text { (\%) }\end{array}$ & $\begin{array}{c}\text { Stroke } \\
(\%)\end{array}$ & ALT>3ALN & $\begin{array}{c}\text { ALT>3ALN } \\
\text { And bili } \\
>2 A L N\end{array}$ & $\begin{array}{l}\text { Refer- } \\
\text { ence }\end{array}$ \\
\hline Api $2.5 \mathrm{mg}$ bid & & 87 & & 7.8 & 1.3 & 1.3 & 0.7 & 2.6 & 0 & [26] \\
\hline Enox $30 \mathrm{mg}$ bid & & 87 & & 6.7 & 0.7 & 0 & 0 & 2.7 & 0 & \\
\hline War INR 1.8-3 & & 89 & & 6.0 & 2.0 & 0.7 & 0 & 2.0 & 0.7 & \\
\hline Api $2.5 \mathrm{mg}$ bid & & & & 8.5 & 1.3 & 0.1 & 0 & 1.0 & 0 & {$[27]$} \\
\hline Enox $30 \mathrm{mg}$ bid & & & & 8.6 & 0.7 & 0.3 & 0.1 & 1.6 & 0.1 & \\
\hline & & & & & 2.0 & & & & & \\
\hline Api $2.5 \mathrm{mg}$ bid & 3 & 52 & 14 & 5 & & 0.1 & 0.1 & 2 & 0.2 & {$[28]$} \\
\hline Enox 40 mg qd & 3 & 55 & 14 & 6 & & 0.1 & 0 & 1 & 0.1 & \\
\hline Api $2.5 \mathrm{mg}$ bid & 3.4 & 65 & & 6.9 & & 0.2 & $<0.1$ & 1.3 & 0.3 & [29] \\
\hline Enox 40 mg qd & 4.2 & 68 & & 6.5 & & 0.2 & 0.2 & 1.5 & 0.1 & \\
\hline
\end{tabular}




\begin{tabular}{|c|c|c|c|c|c|c|c|c|c|c|}
\hline \multicolumn{11}{|l|}{$\begin{array}{l}\text { Dabi } 150 \mathrm{mg} \text { bid } \\
\text { Dabi } 225 \mathrm{mg} \text { bid } \\
\text { Enox } 40 \mathrm{mg} \text { qd }\end{array}$} \\
\hline Dabi 150 mg qd & 3.7 & & & & & 1.0 & & 3.7 & & [31] \\
\hline Dabi 220 mg qd & 3.7 & & & & & 0.4 & & 3.7 & & \\
\hline Enox $40 \mathrm{mg} \mathrm{qd}$ & 4.6 & & & & & 0.5 & & 4.6 & & \\
\hline Dabi 150 mg qd & 8 & 77 & & 8 & & & & 3 & & [32] \\
\hline Dabi 220 mg qd & 6 & 77 & & 8 & & & & 3 & & \\
\hline Enox $40 \mathrm{mg} \mathrm{qd}$ & 6 & 77 & & 7 & & & & 5 & & \\
\hline Dabi 220 mg qd & 5.9 & 68 & 9.1 & 5.6 & & $<0.1$ & 0 & 3.8 & 0.2 & [33] \\
\hline Enox $40 \mathrm{mg} \mathrm{qd}$ & 5.2 & 69 & 9.5 & 5.9 & & $<0.1$ & 0 & 5.6 & 0 & \\
\hline Dabi 150 mg qd & 5 & & & & & & & 0.7 & & [34] \\
\hline Dabi 220 mg qd & 5 & & & & & & & 1.0 & & \\
\hline Enox $30 \mathrm{mg}$ bid & 6 & & & & & & & 0.9 & & \\
\hline Edo $15 \mathrm{mg}$ qd & 1.6 & 35 & 8.9 & 4.2 & & & & 4.2 & 0.5 & [35] \\
\hline Edo $30 \mathrm{mg} \mathrm{qd}$ & 0.6 & 28 & 4.7 & 2.9 & & & & 2.4 & 0.6 & \\
\hline Dalte $5000 \mathrm{IU}$ qd & 1.2 & 36 & 8.1 & 1.7 & & & & 2.9 & 0.6 & \\
\hline Edo $30 \mathrm{mg}$ qd & & & & & & & & 0.6 & 0 & [36] \\
\hline Enox $20 \mathrm{mg} \mathrm{bid}$ & & & & & & & & 5.7 & 0.3 & \\
\hline Edo $15 \mathrm{mg} \mathrm{qd}$ & & 65 & 18 & 0 & & & & 0.4 & & [37] \\
\hline Edo $30 \mathrm{mg}$ qd & & 71 & 26 & 0 & & & & 0.3 & & \\
\hline Enox $20 \mathrm{mg}$ bid & & 83 & 53 & 1.1 & & & & 1.2 & & \\
\hline Edo $30 \mathrm{mg}$ qd & & 65 & & & & & & 2.6 & & [38] \\
\hline Enox $20 \mathrm{mg}$ bid & & 77 & & & & & & 10 & & \\
\hline Riva $10 \mathrm{mg} \mathrm{qd}$ & & 64 & 12 & & 0.4 & 0.1 & 0.1 & 2.0 & 0.1 & [39] \\
\hline Enox $40 \mathrm{mg} \mathrm{qd}$ & & 65 & 12 & & 0.4 & 0.3 & 0.1 & 2.7 & 0.1 & \\
\hline Riva $10 \mathrm{mg}$ qd & 3.8 & 60 & 1.1 & 7.3 & 0.7 & 0.3 & 0.2 & 1.6 & 0.2 & [40] \\
\hline Enox $40 \mathrm{mg} \mathrm{qd}$ & 5.3 & 62 & 1.4 & 10.7 & 0.5 & 0.2 & 0.1 & 4.7 & 0.3 & \\
\hline Riva $10 \mathrm{mg}$ qd & & & 12.013 .0 & & 0.6 & 0.1 & 0.2 & & & [41] \\
\hline Enox $40 \mathrm{mg} \mathrm{qd}$ & & & & & 0.9 & 0.2 & 0 & & & \\
\hline Riva 10 mg qd & & 80 & 20 & 5.2 & 0.3 & 0.1 & 0.1 & 1.3 & 0.1 & [42] \\
\hline Enox $30 \mathrm{mg}$ bid & & 81 & 20 & 7.0 & 0.2 & 0 & 0 & 2.6 & 0.2 & \\
\hline Riva $10 \mathrm{mg}$ qd & & & & & & & & & & [43] \\
\hline Aspirin $81 \mathrm{mg} \mathrm{qd}$ & & & & & & & & & & \\
\hline
\end{tabular}

Table 4: Comparative studies in orthopaedic surgery, safety results.

\section{AE: Adverse Events}

AE-S: Severe Adverse Events

MI: Myocardial Infarction. 
152

\begin{tabular}{|c|c|c|c|c|c|c|c|c|c|c|}
\hline Medicines & $\begin{array}{c}\text { Major } \\
\text { bleed- } \\
\text { ing } \\
(\%)\end{array}$ & $\begin{array}{c}\text { Overt } \\
\text { bleed- } \\
\text { ing } \\
(\%)\end{array}$ & $\begin{array}{c}\text { Minor } \\
\text { bleed- } \\
\text { ing } \\
(\%)\end{array}$ & $\begin{array}{c}\text { Non-major } \\
\text { bleeding } \\
(\%)\end{array}$ & $\begin{array}{c}\text { All bleed- } \\
\text { ing (\%) }\end{array}$ & $\begin{array}{c}\text { Bleeding } \\
\text { with surgical } \\
\text { intervention } \\
(\%)\end{array}$ & $\begin{array}{c}\text { Fatal } \\
\text { bleeding } \\
(\%)\end{array}$ & $\begin{array}{c}\text { Bleeding } \\
\text { at critical } \\
\text { site } \\
(\%)\end{array}$ & $\begin{array}{c}\text { Bleeding } \\
\text { at surgi- } \\
\text { cal site } \\
\text { (\%) }\end{array}$ & $\begin{array}{c}\text { Ref- } \\
\text { er- } \\
\text { ence }\end{array}$ \\
\hline Api $2.5 \mathrm{mg}$ bid & 0 & & 3.9 & & 3.0 & 0 & & & & {$[26]$} \\
\hline Enox $30 \mathrm{mg}$ bid & 0 & & 4.0 & & 4.0 & 0 & & & & \\
\hline War INR 1.8-3 & 0 & & 5.3 & & 5.3 & 0 & & & & \\
\hline Api $2.5 \mathrm{mg}$ bid & 0.7 & 0.6 & 2.4 & & 5.3 & & 0 & 0 & 0.5 & [27] \\
\hline Enox $30 \mathrm{mg}$ bid & 1.4 & 1.4 & 2.5 & & 6.8 & & $<0.1$ & 0.1 & 0.9 & \\
\hline Api $2.5 \mathrm{mg}$ bid & 0.6 & 0.5 & 3.4 & & 6.9 & & & 0 & 0.5 & {$[28]$} \\
\hline Enox $40 \mathrm{mg}$ qd & 0.9 & 0.9 & 3.6 & & 8.4 & & & 0 & 0.7 & \\
\hline Api $2.5 \mathrm{mg}$ bid & 0.8 & & 6.9 & & 11.7 & $<0.1$ & 0 & 0 & 0.7 & [29] \\
\hline Enox $40 \mathrm{mg}$ qd & 0.7 & & 7.3 & & 12.6 & $<0.1$ & 0 & 0 & 0.6 & \\
\hline Dabi $150 \mathrm{mg}$ bid & 4.1 & & 7.9 & & 12.0 & & & & & {$[30]$} \\
\hline Dabi 225 mg bid & 3.8 & & 9.7 & & 13.5 & & & & & \\
\hline Enox $40 \mathrm{mg}$ qd & 2.0 & & 6.4 & & 8.4 & & & & & \\
\hline Dabi $150 \mathrm{mg}$ qd & 1.3 & & 8.4 & 6.8 & & 0.1 & 0 & 0.1 & & {$[31]$} \\
\hline Dabi 220 mg qd & 1.5 & & 8.8 & 5.9 & & 0.2 & 0 & 0 & & \\
\hline Enox $40 \mathrm{mg}$ qd & 1.3 & & 9.9 & 5.3 & & 0.1 & 0 & 0 & & \\
\hline Dabi 150 mg qd & 1.3 & & 6.2 & 4.7 & & 0.3 & 0.1 & 0 & & {$[32]$} \\
\hline Dabi 220 mg qd & 2.0 & & 6.1 & 4.2 & & 0.2 & 0.1 & 0 & & \\
\hline Enox $40 \mathrm{mg} \mathrm{qd}$ & 1.6 & & 6.4 & 3.5 & & 0.3 & 0 & 0 & & \\
\hline Dabi 220 mg qd & 1.4 & 1.3 & 6.0 & 2.3 & 9.7 & 0 & 0 & 0.1 & & [33] \\
\hline Enox $40 \mathrm{mg}$ qd & 0.9 & 0.7 & 5.4 & 2.0 & 8.3 & 0 & 0 & 0 & & \\
\hline Dabi 150 mg qd & 0.6 & & & 2.5 & & 0 & 0 & & 0.2 & {$[34]$} \\
\hline Dabi $220 \mathrm{mg}$ qd & 0.6 & & & 2.7 & & 0 & 0 & & 0.3 & \\
\hline Enox $40 \mathrm{mg}$ qd & 1.4 & & & 2.4 & & 0.1 & 0 & & 1.4 & \\
\hline Edo $15 \mathrm{mg}$ qd & 0.5 & & & 1.2 & 2.1 & & & & & [35] \\
\hline Edo $30 \mathrm{mg}$ qd & 0.6 & & & 1.0 & 1.8 & & & & & \\
\hline Dalte $5000 \mathrm{IU}$ qd & 0 & & & 1 & 0.6 & & & & & \\
\hline Edo $30 \mathrm{mg} \mathrm{qd}$ & 1.1 & & & 5.1 & 6.2 & & & & & {$[36]$} \\
\hline Enox $20 \mathrm{mg}$ bid & 0.3 & & & 3.4 & $\begin{array}{l}3.7 \\
\\
\text { (Major } \\
+ \text { non } \\
\text { major) }\end{array}$ & & & & & \\
\hline
\end{tabular}




\begin{tabular}{|c|c|c|c|c|c|c|c|c|c|}
\hline $\begin{array}{l}\text { Edo } 15 \mathrm{mg} \text { qd } \\
\text { Edo } 30 \mathrm{mg} \text { qd } \\
\text { Enox } 20 \mathrm{mg} \text { bid }\end{array}$ & $\begin{array}{c}0 \\
1.2 \\
0\end{array}$ & & & $\begin{array}{c}2.2 \\
1.2 \\
2.3 \\
\text { (Major + } \\
\text { non major) }\end{array}$ & $\begin{array}{l}18 \\
21 \\
22\end{array}$ & & & & [37] \\
\hline $\begin{array}{l}\text { Edo } 30 \mathrm{mg} \text { qd } \\
\text { Enox } 20 \mathrm{mg} \text { bid }\end{array}$ & $\begin{array}{l}0.7 \\
2.0\end{array}$ & & $\begin{array}{l}18.8 \\
13.0\end{array}$ & $\begin{array}{l}2.0 \\
1.7\end{array}$ & $\begin{array}{l}20.5 \\
15.9\end{array}$ & & & & [38] \\
\hline $\begin{array}{l}\text { Riva } 10 \mathrm{mg} \text { qd } \\
\text { Enox } 40 \mathrm{mg} \text { qd }\end{array}$ & $\begin{array}{l}0.3 \\
0.1 \\
\end{array}$ & $\begin{array}{l}0.2 \\
0.1 \\
\end{array}$ & & $\begin{array}{l}5.8 \\
5.8 \\
\end{array}$ & & $\begin{array}{l}0.1 \\
0.1 \\
\end{array}$ & $\begin{array}{c}0.1 \\
0 \\
\end{array}$ & $\begin{array}{c}0.1 \\
0 \\
0\end{array}$ & [39] \\
\hline $\begin{array}{l}\text { Riva } 10 \mathrm{mg} \text { qd } \\
\text { Enox } 40 \mathrm{mg} \mathrm{qd}\end{array}$ & $\begin{array}{l}0.1 \\
0.1 \\
\end{array}$ & $\begin{array}{c}0.1 \\
0 \\
\end{array}$ & & $\begin{array}{l}3.3 \\
2.7\end{array}$ & $\begin{array}{l}4.7 \\
4.1 \\
\end{array}$ & $\begin{array}{l}0 \\
0 \\
\end{array}$ & $\begin{array}{l}0 \\
0 \\
\end{array}$ & $\begin{array}{c}0 \\
0.1 \\
\end{array}$ & [40] \\
\hline $\begin{array}{l}\text { Riva } 10 \mathrm{mg} \text { qd } \\
\text { Enox } 40 \mathrm{mg} \text { qd }\end{array}$ & $\begin{array}{l}0.6 \\
0.5 \\
\end{array}$ & $\begin{array}{c}0.2 \\
0 \\
\end{array}$ & & $\begin{array}{l}2.7 \\
2.3 \\
\end{array}$ & & $\begin{array}{l}0.4 \\
0.3 \\
\end{array}$ & $\begin{array}{l}0 \\
0\end{array}$ & $\begin{array}{c}0 \\
0.1 \\
\end{array}$ & [41] \\
\hline $\begin{array}{l}\text { Riva } 10 \text { mg qd } \\
\text { Enox } 30 \text { mg bid }\end{array}$ & $\begin{array}{l}0.7 \\
0.3 \\
\end{array}$ & $\begin{array}{c}0.3 \\
0 \\
\end{array}$ & & $\begin{array}{l}2.6 \\
2.0 \\
\end{array}$ & & $\begin{array}{l}0.3 \\
0.1 \\
\end{array}$ & $\begin{array}{c}0.1 \\
0 \\
\end{array}$ & $\begin{array}{l}0.1 \\
0.1\end{array}$ & [42] \\
\hline $\begin{array}{l}\text { Riva } 10 \mathrm{mg} \text { qd } \\
\text { Aspirin } 81 \mathrm{mg} \text { qd }\end{array}$ & $\begin{array}{l}0.3 \\
0.6 \\
\end{array}$ & & & & $\begin{array}{l}1.2 \\
1.4 \\
\end{array}$ & & & & [43] \\
\hline
\end{tabular}

Table 5: Comparative studies in orthopaedic surgery, safety results: bleeding.

\begin{tabular}{|c|c|c|c|c|c|c|c|c|c|c|c|}
\hline Medicines & $\mathbf{N}$ & Age & $\begin{array}{c}\text { Female } \\
(\%)\end{array}$ & Inclusion & $\begin{array}{c}\text { Blood } \\
\text { pressure } \\
\text { S/D }\end{array}$ & BMI & Design & \begin{tabular}{|c}
$\begin{array}{c}\text { Duration of } \\
\text { follow-up }\end{array}$ \\
(years) \\
\end{tabular} & $\begin{array}{l}\text { CHAD } \\
\text { score } \\
\text { mean }\end{array}$ & $\begin{array}{l}\text { End- } \\
\text { point }\end{array}$ & Ref \\
\hline $\begin{array}{l}\text { Api } 5 \text { mg bid } \\
\text { Aspirin 81-324 } \\
\text { mg qd }\end{array}$ & $\begin{array}{l}2808 \\
2791\end{array}$ & $\begin{array}{l}70 \\
70\end{array}$ & $\begin{array}{l}41 \\
42\end{array}$ & $\begin{array}{l}\text { AF, at risk for } \\
\text { stroke, not suit- } \\
\text { able for vit K } \\
\text { antagonist }\end{array}$ & $\begin{array}{l}132 \\
132\end{array}$ & $\begin{array}{l}28 \\
28\end{array}$ & Open & 1.1 & $\begin{array}{l}2.0 \\
2.1\end{array}$ & $\begin{array}{l}\text { Stroke } \\
\text { or SE }\end{array}$ & [44] \\
\hline $\begin{array}{l}\text { Api } 5 \text { mg bid } \\
\text { Warfarin INR 2-3 }\end{array}$ & $\begin{array}{l}9120 \\
9081 \\
\end{array}$ & $\begin{array}{l}70 \\
70 \\
\end{array}$ & $\begin{array}{l}36 \\
35 \\
\end{array}$ & $\begin{array}{l}\mathrm{AF}+\text { at least one } \\
\text { risk factor for } \\
\text { stroke }\end{array}$ & $\begin{array}{l}130 \\
130 \\
\end{array}$ & & Open & 1.8 & $\begin{array}{l}2.1 \\
2.1\end{array}$ & $\begin{array}{l}\text { Stroke } \\
\text { or SE }\end{array}$ & [45] \\
\hline $\begin{array}{l}\text { Dab } 110 \text { mg bid } \\
\text { Dab } 150 \text { mg bid } \\
\text { Warfarin INR 2-3 }\end{array}$ & $\begin{array}{l}6015 \\
6076 \\
6022\end{array}$ & $\begin{array}{l}71 \\
71 \\
72\end{array}$ & $\begin{array}{l}36 \\
37 \\
37\end{array}$ & $\begin{array}{l}\mathrm{AF} \text {, at risk for } \\
\text { stroke }\end{array}$ & $\begin{array}{l}131 \\
131 \\
131\end{array}$ & & $\begin{array}{c}\text { DB } \\
\text { DB } \\
\text { Open }\end{array}$ & 2.0 & $\begin{array}{l}2.1 \\
2.2 \\
2.1\end{array}$ & $\begin{array}{l}\text { Stroke } \\
\text { or SE }\end{array}$ & [46] \\
\hline $\begin{array}{l}\text { Dab } 150 \text { mg bid } \\
\text { Warfarin INR 2-3 }\end{array}$ & $\begin{array}{l}317 \\
318\end{array}$ & $\begin{array}{l}59 \\
59\end{array}$ & $\begin{array}{l}27 \\
22\end{array}$ & $\mathrm{AF}$, ablation & $\begin{array}{l}131 \\
131 \\
131\end{array}$ & $\begin{array}{l}29 \\
29\end{array}$ & Open & 8 weeks & $\begin{array}{l}2.0 \\
2.2\end{array}$ & $\begin{array}{l}\text { Bleed- } \\
\text { ing }\end{array}$ & [47] \\
\hline $\begin{array}{l}\text { Dab } 110 \text { mg bid } \\
\text { Warfarin/Aspirin } \\
\text { Dab } 150 \text { mg bid } \\
\text { Warfarin/Aspirin }\end{array}$ & $\begin{array}{l}981 \\
981 \\
763 \\
764\end{array}$ & $\begin{array}{l}72 \\
72 \\
69 \\
69\end{array}$ & $\begin{array}{l}26 \\
23 \\
22 \\
22\end{array}$ & $\begin{array}{c}\text { AF, PCI } \\
\text { Combi with } \\
\text { ticagrelor or } \\
\text { clopidogrel }\end{array}$ & & & Open & 14 months & $\begin{array}{l}3.7 \\
3.8 \\
3.3 \\
3.6\end{array}$ & $\begin{array}{l}\text { MI, } \\
\text { stroke, } \\
\text { SE }\end{array}$ & [48] \\
\hline
\end{tabular}




\begin{tabular}{|c|c|c|c|c|c|c|c|c|c|c|c|}
\hline $\begin{array}{l}\text { Edo } 30 \mathrm{mg} \text { qd } \\
\text { Edo } 60 \mathrm{mg} \text { qd } \\
\text { Warfarin INR 2-3 }\end{array}$ & $\begin{array}{l}7034 \\
7035 \\
7036\end{array}$ & $\begin{array}{l}72 \\
72 \\
72\end{array}$ & $\begin{array}{l}39 \\
38 \\
38\end{array}$ & $\begin{array}{l}\text { AF, moderate } \\
\text { to high risk for } \\
\text { stroke }\end{array}$ & & & $\mathrm{DB}, \mathrm{DD}$ & 2.8 & $\begin{array}{l}2.8 \\
2.8 \\
2.8\end{array}$ & $\begin{array}{l}\text { Stroke } \\
\text { or SE }\end{array}$ & [49] \\
\hline $\begin{array}{l}\text { Riv } 20 \text { mg qd } \\
\text { Warfarin INR 2-3 }\end{array}$ & $\begin{array}{l}7131 \\
7133\end{array}$ & $\begin{array}{l}73 \\
73\end{array}$ & $\begin{array}{l}40 \\
40\end{array}$ & $\begin{array}{l}\text { AF, moderate } \\
\text { to high risk for } \\
\text { stroke }\end{array}$ & $\begin{array}{l}130 \\
130\end{array}$ & $\begin{array}{l}28 \\
28\end{array}$ & $\mathrm{DB}, \mathrm{DD}$ & 2.5 & $\begin{array}{l}3.5 \\
3.5\end{array}$ & $\begin{array}{l}\text { Stroke } \\
\text { or SE }\end{array}$ & [50] \\
\hline $\begin{array}{l}\text { Riv } 20 \text { mg qd } \\
\text { Vit K antag INR } \\
2-3\end{array}$ & $\begin{array}{l}1002 \\
502\end{array}$ & $\begin{array}{l}65 \\
65\end{array}$ & $\begin{array}{l}27 \\
27\end{array}$ & $\begin{array}{l}\text { AF, cardiover- } \\
\text { sion }\end{array}$ & & $\begin{array}{l}30 \\
30\end{array}$ & Ope & 8 weeks & $\begin{array}{l}38 \% \\
39 \% \\
(>2)\end{array}$ & $\begin{array}{l}\text { Stroke, } \\
\text { TIA, } \\
\text { PE, MI, } \\
\text { CV-D }\end{array}$ & [51] \\
\hline
\end{tabular}

Table 6: Comparative studies in atrial fibrillation, baseline data and design.

AF: Atrial Fibrillation

AF-NV: Non Valvular Atrial Fibrillation

CV-D: Cardiovascular Death

I-S: Ischaemic Stroke

MI: Myocardial Infarction

PE: Peripheral Embolism

SE: Systemic Embolism.

\begin{tabular}{|c|c|c|c|c|c|c|c|c|}
\hline Medicines & $\begin{array}{l}\text { Type of AF } \\
\text { (\%) }\end{array}$ & & & $\begin{array}{c}\text { Previous } \\
\text { stroke (\%) }\end{array}$ & $\begin{array}{c}\text { Heart } \\
\text { failure (\%) }\end{array}$ & Diabetes & Hypertension & Ref \\
\hline & Persistent & Paroxysmal & Permanent & & & & & \\
\hline Api $5 \mathrm{mg}$ bid & 21 & 27 & 52 & 14 & 40 & 19 & 86 & [44] \\
\hline Aspirin 81-324 mg qd & 21 & 27 & 52 & 13 & 38 & 20 & 87 & \\
\hline Api $5 \mathrm{mg}$ bid & 85 & 15 & & 19 & 36 & 25 & 87 & {$[45]$} \\
\hline Warfarin INR 2-3 & 84 & 16 & & 20 & 35 & 25 & 88 & \\
\hline Dab $110 \mathrm{mg}$ bid & 32 & 32 & 35 & 20 & 32 & 23 & 79 & {$[46]$} \\
\hline Dab 150 mg bid & 31 & 33 & 36 & 20 & 32 & 23 & 79 & \\
\hline Warfarin INR 2-3 & 32 & 34 & 34 & 20 & 32 & 23 & 79 & \\
\hline Dab 150 mg bid & 27 & 67 & 6 & 3 & 10 & 10 & 52 & [47] \\
\hline Warfarin INR 2-3 & 26 & 69 & 6 & 3 & 11 & 11 & 56 & \\
\hline Dab 110 mg bid & 18 & 50 & 33 & 8 & & 37 & & {$[48]$} \\
\hline Warfarin/Aspirin & 18 & 49 & 32 & 10 & & 38 & & \\
\hline Dab 150 mg bid & 17 & 50 & 33 & 7 & & 34 & & \\
\hline Warfarin/Aspirin & 20 & 49 & 31 & 10 & & 40 & & \\
\hline Edo 30 mg qd & & 26 & & 29 & 57 & 36 & 94 & [49] \\
\hline Edo $60 \mathrm{mg} \mathrm{qd}$ & & 25 & & 28 & 58 & 36 & 94 & \\
\hline Warfarin INR 2-3 & & 25 & & 28 & 58 & 36 & 94 & \\
\hline Riv 20 mg qd & 81 & 18 & & 55 & 63 & 40 & 90 & {$[50]$} \\
\hline Warfarin INR 2-3 & 81 & 18 & & 55 & 62 & 40 & 91 & \\
\hline Riv 20 mg qd & 56 & 17 & 3 & 3 & 20 & 20 & 65 & {$[51]$} \\
\hline Vit K antag INR 2-3 & 50 & 23 & 5 & 4 & 15 & 21 & 69 & \\
\hline
\end{tabular}

Table 7: Comparative studies in atrial fibrillation, baseline data and design. 


\begin{tabular}{|c|c|c|c|c|c|c|c|c|}
\hline Medicines & $\begin{array}{c}\text { ACE/ARB } \\
\%\end{array}$ & $\begin{array}{c}\text { Calcium antagonist } \\
\%\end{array}$ & $\begin{array}{c}\text { Beta blocker } \\
\%\end{array}$ & $\begin{array}{c}\text { Digoxin } \\
\%\end{array}$ & $\begin{array}{c}\text { Amiodarone } \\
\%\end{array}$ & $\begin{array}{c}\text { Statin } \\
\%\end{array}$ & $\begin{array}{c}\text { Aspirin } \\
\%\end{array}$ & Ref \\
\hline $\begin{array}{l}\text { Api } 5 \mathrm{mg} \text { bid } \\
\text { Aspirin } 81-324 \mathrm{mg} \mathrm{qd}\end{array}$ & $\begin{array}{l}64 \\
64\end{array}$ & $\begin{array}{l}9 \\
9\end{array}$ & $\begin{array}{l}56 \\
55\end{array}$ & $\begin{array}{l}29 \\
27\end{array}$ & $\begin{array}{l}11 \\
12\end{array}$ & $\begin{array}{l}31 \\
31\end{array}$ & $\begin{array}{c}0 \\
100\end{array}$ & [44] \\
\hline $\begin{array}{l}\text { Api } 5 \text { mg bid } \\
\text { Warfarin INR 2-3 }\end{array}$ & $\begin{array}{l}71 \\
70\end{array}$ & $\begin{array}{l}30 \\
31\end{array}$ & $\begin{array}{l}64 \\
63\end{array}$ & $\begin{array}{l}32 \\
32\end{array}$ & $\begin{array}{l}11 \\
12\end{array}$ & $\begin{array}{l}45 \\
45\end{array}$ & $\begin{array}{l}31 \\
31\end{array}$ & [45] \\
\hline $\begin{array}{l}\text { Dab } 110 \text { mg bid } \\
\text { Dab } 150 \text { mg bid } \\
\text { Warfarin INR 2-3 }\end{array}$ & $\begin{array}{l}66 \\
67 \\
66\end{array}$ & & $\begin{array}{l}63 \\
64 \\
62\end{array}$ & & $\begin{array}{l}10 \\
11 \\
11\end{array}$ & $\begin{array}{l}45 \\
44 \\
45\end{array}$ & $\begin{array}{l}40 \\
39 \\
41\end{array}$ & [46] \\
\hline $\begin{array}{l}\text { Dab } 150 \text { mg bid } \\
\text { Warfarin INR 2-3 }\end{array}$ & & & $\begin{array}{l}58 \\
60\end{array}$ & & & $\begin{array}{l}31 \\
30\end{array}$ & & [47] \\
\hline $\begin{array}{l}\text { Edo } 30 \mathrm{mg} \text { qd } \\
\text { Edo } 60 \mathrm{mg} \text { qd } \\
\text { Warfarin INR 2-3 }\end{array}$ & & & & $\begin{array}{l}30 \\
30 \\
31\end{array}$ & $\begin{array}{l}11 \\
12 \\
12\end{array}$ & & $\begin{array}{l}29 \\
29 \\
30\end{array}$ & [49] \\
\hline $\begin{array}{l}\text { Riv } 20 \text { mg qd } \\
\text { Warfarin INR 2-3 }\end{array}$ & $\begin{array}{l}55 \\
54\end{array}$ & & $\begin{array}{l}65 \\
65\end{array}$ & $\begin{array}{l}39 \\
39\end{array}$ & & $\begin{array}{l}43 \\
43\end{array}$ & $\begin{array}{l}36 \\
37\end{array}$ & [50] \\
\hline $\begin{array}{l}\text { Riv } 20 \text { mg qd } \\
\text { Vit K antag INR 2-3 }\end{array}$ & & & & & & & $\begin{array}{l}27 \\
28\end{array}$ & [51] \\
\hline
\end{tabular}

Table 8: Comparative studies in atrial fibrillation, baseline data and design.

\begin{tabular}{|c|c|c|c|c|c|c|c|c|}
\hline Medicines & $\begin{array}{c}\text { Stroke } \\
\quad \%\end{array}$ & $\begin{array}{c}\text { Stroke } \\
\text { ischaemic or } \\
\text { unspecified } \\
\% \\
\%\end{array}$ & $\begin{array}{c}\text { Stroke } \\
\text { Hemorrhagic } \\
\%\end{array}$ & $\begin{array}{c}\text { Stroke dis- } \\
\text { abling or fatal } \\
\%\end{array}$ & $\begin{array}{l}\text { SE } \\
\%\end{array}$ & $\begin{array}{c}\text { Stroke or SE } \\
\%\end{array}$ & $\begin{array}{l}\text { TIA } \\
\%\end{array}$ & Ref \\
\hline $\begin{array}{l}\text { Api } 5 \mathrm{mg} \text { bid } \\
\text { Aspirin 81-324 mg qd }\end{array}$ & $\begin{array}{l}1.6 / y r \\
3.4 / y r \\
p<0.001\end{array}$ & $\begin{array}{c}1.1 / \mathrm{yr} \\
3.0 / \mathrm{yr} \\
\mathrm{p}<0.001\end{array}$ & $\begin{array}{l}0.2 / \mathrm{yr} \\
0.3 / \mathrm{yr}\end{array}$ & $\begin{array}{c}1.0 / \mathrm{yr} \\
2.3 / \mathrm{yr} \\
\mathrm{p}<0.001\end{array}$ & $\begin{array}{l}0.1 / \mathrm{yr} \\
0.4 / \mathrm{yr}\end{array}$ & $\begin{array}{c}1.6 / \mathrm{yr} \\
3.7 / \mathrm{yr} \\
\mathrm{P}<0.001\end{array}$ & & [44] \\
\hline $\begin{array}{l}\text { Api } 5 \text { mg bid } \\
\text { Warfarin INR 2-3 }\end{array}$ & $\begin{array}{l}1.19 / \mathrm{yr} \\
1.51 / \mathrm{yr} \\
\mathrm{P}=0.01\end{array}$ & $\begin{array}{l}0.97 / \mathrm{yr} \\
1.05 / \mathrm{yr}\end{array}$ & $\begin{array}{l}0.24 / \mathrm{yr} \\
0.47 / \mathrm{yr} \\
\mathrm{P}<0.001\end{array}$ & & $\begin{array}{l}0.09 / \mathrm{yr} \\
0.10 / \mathrm{yr}\end{array}$ & $\begin{array}{l}1.27 / \mathrm{yr} \\
1.60 / \mathrm{yr} \\
\mathrm{P}=0.01\end{array}$ & & [45] \\
\hline $\begin{array}{l}\text { Dab } 110 \text { mg bid } \\
\text { Dab } 150 \text { mg bid } \\
\text { Warfarin INR 2-3 }\end{array}$ & $\begin{array}{l}1.44 / \mathrm{yr} \\
1.01 / \mathrm{yr} \\
1.57 / \mathrm{yr}\end{array}$ & $\begin{array}{l}1.34 / \mathrm{yr} \\
0.92 / \mathrm{yr} \\
1.20 / \mathrm{yr}\end{array}$ & $\begin{array}{l}0.12 / \mathrm{yr} \\
0.10 / \mathrm{yr} \\
0.38 / \mathrm{yr} \\
\mathrm{P}<0.001\end{array}$ & $\begin{array}{l}0.94 / \mathrm{yr} \\
0.66 / \mathrm{yr} \\
1.00 / \mathrm{yr}\end{array}$ & & $\begin{array}{c}1.53 / \mathrm{yr} \\
1.11 / \mathrm{yr} \\
1.69 / \mathrm{yr} \\
\text { NI p=0.02 for } \\
150 \mathrm{mg} \text { vs } \\
\text { warfarin }\end{array}$ & & [46] \\
\hline
\end{tabular}






Table 9: Comparative studies in atrial fibrillation, results.

\begin{tabular}{|c|c|c|c|c|c|c|c|}
\hline Medicines & MI & $\begin{array}{l}\text { CV hospital- } \\
\text { ization }\end{array}$ & $\begin{array}{c}\text { Death } \\
\text { CV }\end{array}$ & $\begin{array}{l}\text { Death } \\
\text { total }\end{array}$ & $\begin{array}{c}\text { Composite } \\
\text { Stroke, SE, death }\end{array}$ & $\begin{array}{c}\text { Composite } \\
\text { Stroke, SE, MI, death }\end{array}$ & Ref \\
\hline $\begin{array}{l}\text { Api } 5 \mathrm{mg} \text { bid } \\
\text { Aspirin } 81-324 \mathrm{mg} \mathrm{qd}\end{array}$ & $\begin{array}{l}0.8 \\
0.9\end{array}$ & $\begin{array}{c}12.6 \\
15.9 \\
\mathrm{P}<0.001\end{array}$ & $\begin{array}{l}2.7 \\
3.1\end{array}$ & $\begin{array}{l}3.5 \\
4.4\end{array}$ & $\begin{array}{c}4.6 \\
7.2 \\
\mathrm{P}<0.001\end{array}$ & & [44] \\
\hline $\begin{array}{l}\text { Api } 5 \text { mg bid } \\
\text { Warfarin INR 2-3 }\end{array}$ & $\begin{array}{l}0.53 / \mathrm{yr} \\
0.61 / \mathrm{yr}\end{array}$ & & & $\begin{array}{l}3.52 / \mathrm{yr} \\
3.94 / \mathrm{yr} \\
\mathrm{P}<0.05\end{array}$ & $\begin{array}{l}4.49 / \mathrm{yr} \\
5.05 / \mathrm{yr} \\
\mathrm{P}=0.02\end{array}$ & $\begin{array}{l}4.85 / \mathrm{yr} \\
5.49 / \mathrm{yr} \\
\mathrm{P}=0.01\end{array}$ & [45] \\
\hline $\begin{array}{l}\text { Dab } 110 \text { mg bid } \\
\text { Dab } 150 \text { mg bid } \\
\text { Warfarin INR 2-3 }\end{array}$ & $\begin{array}{l}0.72 / \mathrm{yr} \\
0.74 / \mathrm{yr} \\
0.53 / \mathrm{yr}\end{array}$ & & $\begin{array}{l}2.43 / \mathrm{yr} \\
2.28 / \mathrm{yr} \\
2.69 / \mathrm{yr}\end{array}$ & $\begin{array}{l}3.75 / \mathrm{yr} \\
3.64 / \mathrm{yr} \\
4.31 / \mathrm{yr}\end{array}$ & & & [46] \\
\hline $\begin{array}{l}\text { Dab } 150 \text { mg bid } \\
\text { Warfarin INR 2-3 }\end{array}$ & & & & & & & [47] \\
\hline
\end{tabular}




\begin{tabular}{|c|c|c|c|c|c|}
\hline $\begin{array}{l}\text { Dab } 110 \text { mg bid } \\
\text { Warfarin/Aspirin } \\
\text { Dab } 150 \text { mg bid } \\
\text { Warfarin/Aspirin }\end{array}$ & $\begin{array}{l}4.5 \% \\
3.0 \% \\
3.4 \% \\
2.9 \%\end{array}$ & & $\begin{array}{l}5.6 \% \\
4.9 \% \\
3.9 \% \\
4.6 \%\end{array}$ & & [48] \\
\hline $\begin{array}{l}\text { Edo } 30 \mathrm{mg} \text { qd } \\
\text { Edo } 60 \mathrm{mg} \text { qd } \\
\text { Warfarin INR 2-3 }\end{array}$ & $\begin{array}{c}0.89 / \mathrm{yr} \\
0.70 / \mathrm{yr} \\
0.75 / \mathrm{y}\end{array}$ & $\begin{array}{l}2.71 / \mathrm{yr} \\
2.74 / \mathrm{yr} \\
3.17 / \mathrm{yr} \\
\mathrm{P}=0.013\end{array}$ & $\begin{array}{l}3.80 / \mathrm{yr} \\
3.99 / \mathrm{yr} \\
4.35 / \mathrm{yr}\end{array}$ & $\begin{array}{l}5.23 / \mathrm{yr} \\
5.01 / \mathrm{yr} \\
5.57 / \mathrm{yr} \\
\mathrm{P}=0.02\end{array}$ & [49] \\
\hline $\begin{array}{l}\text { Riv } 20 \text { mg qd } \\
\text { Warfarin INR 2-3 }\end{array}$ & $\begin{array}{l}0.9 / \mathrm{yr} \\
1.1 / \mathrm{yr}\end{array}$ & & $\begin{array}{l}1.9 / \mathrm{yr} \\
2.2 / \mathrm{yr}\end{array}$ & & [50] \\
\hline $\begin{array}{l}\text { Riv } 20 \text { mg qd } \\
\text { Vit K antag INR 2-3 }\end{array}$ & $\begin{array}{l}0.10 \\
0.20\end{array}$ & $\begin{array}{l}0.41 \\
0.41\end{array}$ & $\begin{array}{l}0.51 \\
0.61\end{array}$ & & [51] \\
\hline
\end{tabular}

Table 10: Comparative studies in atrial fibrillation, results.

\begin{tabular}{|c|c|c|c|c|c|c|c|}
\hline Medicines & $\begin{array}{c}\text { Withdrawal } \\
\text { AE (\%) }\end{array}$ & AE (\%) & $\begin{array}{l}\text { AE drug re- } \\
\text { lated (\%) }\end{array}$ & $\begin{array}{l}\text { AE-S } \\
(\%)\end{array}$ & ALT $>3$ ALN & $\begin{array}{c}\text { ALT }>3 \text { ALN } \\
\text { And bili }>2 \text { ALN }\end{array}$ & Reference \\
\hline $\begin{array}{l}\text { Api } 5 \text { mg bid } \\
\text { Aspirin 81-324 mg qd }\end{array}$ & & & & $\begin{array}{c}22 \\
27 \\
P<0.001\end{array}$ & $\begin{array}{l}1.4 \\
1.6\end{array}$ & $\begin{array}{l}0.2 \\
0.4\end{array}$ & [44] \\
\hline $\begin{array}{l}\text { Api } 5 \text { mg bid } \\
\text { Warfarin INR 2-3 }\end{array}$ & & $\begin{array}{l}81 \\
83 \\
\end{array}$ & & $\begin{array}{l}35 \\
37 \\
\end{array}$ & $\begin{array}{l}1.1 \\
1.0\end{array}$ & $\begin{array}{l}0.3 \\
0.4 \\
\end{array}$ & [45] \\
\hline $\begin{array}{l}\text { Dab } 110 \text { mg bid } \\
\text { Dab } 150 \text { mg bid } \\
\text { Warfarin INR 2-3 }\end{array}$ & $\begin{array}{l}2.7 \\
2.7 \\
1.7 \\
\end{array}$ & & & & $\begin{array}{l}2.1 \\
1.9 \\
2.2 \\
\end{array}$ & $\begin{array}{l}0.2 \\
0.2 \\
0.3 \\
\end{array}$ & [46] \\
\hline $\begin{array}{l}\text { Dab } 150 \text { mg bid } \\
\text { Warfarin INR 2-3 }\end{array}$ & $\begin{array}{l}2 \\
2\end{array}$ & & & & & & [47] \\
\hline $\begin{array}{l}\text { Dab } 110 \text { mg bid } \\
\text { Warfarin/Aspirin } \\
\text { Dab } 150 \text { mg bid } \\
\text { Warfarin/Aspirin }\end{array}$ & $\begin{array}{l}6 \\
6 \\
6 \\
6\end{array}$ & & & $\begin{array}{l}43 \\
42 \\
40 \\
42\end{array}$ & & & [48] \\
\hline $\begin{array}{l}\text { Edo } 30 \mathrm{mg} \text { qd } \\
\text { Edo } 60 \mathrm{mg} \text { qd } \\
\text { Warfarin INR 2-3 }\end{array}$ & $\begin{array}{l}16 \\
17 \\
17\end{array}$ & $\begin{array}{l}84 \\
84 \\
84 \\
\end{array}$ & $\begin{array}{l}10 \\
11 \\
12 \\
\end{array}$ & $\begin{array}{l}18 \\
17 \\
18\end{array}$ & $\begin{array}{l}2.1 \\
2.2 \\
2.1\end{array}$ & $\begin{array}{l}0.2 \\
0.2 \\
0.1 \\
\end{array}$ & [49] \\
\hline $\begin{array}{l}\text { Riv } 20 \text { mg qd } \\
\text { Warfarin INR 2-3 }\end{array}$ & & $\begin{array}{l}81 \\
82\end{array}$ & & & & $\begin{array}{l}0.5 \\
0.5 \\
\end{array}$ & [50] \\
\hline $\begin{array}{l}\text { Riv } 20 \text { mg qd } \\
\text { Vit K antag INR 2-3 }\end{array}$ & & & & & & & [51] \\
\hline
\end{tabular}

Table 11: Comparative studies in atrial fibrillation, safety results. 


\begin{tabular}{|c|c|c|c|c|c|c|c|c|c|}
\hline Medicines & $\begin{array}{c}\text { Major } \\
\text { bleeding }\end{array}$ & $\begin{array}{c}\text { Intracra- } \\
\text { nial bleed- } \\
\text { ing }\end{array}$ & $\begin{array}{c}\text { Fatal } \\
\text { bleeding }\end{array}$ & $\begin{array}{c}\text { Clinically } \\
\text { relevant } \\
\text { non-major }\end{array}$ & $\begin{array}{c}\text { Major or Clini- } \\
\text { cally relevant } \\
\text { non-major }\end{array}$ & $\begin{array}{c}\text { Minor } \\
\text { bleeding }\end{array}$ & $\begin{array}{l}\text { Any } \\
\text { bleed- } \\
\text { ing }\end{array}$ & $\begin{array}{c}\text { Bleeding } \\
\text { in critical } \\
\text { organ }\end{array}$ & Ref \\
\hline Api $5 \mathrm{mg}$ bid & 1.4 & 0.4 & 0.1 & 3.1 & & 6.3 & & & {$[44]$} \\
\hline $\begin{array}{l}\text { Aspirin 81-324 } \\
\text { mg qd }\end{array}$ & 1.2 & 0.4 & 0.2 & 2.7 & & 5.0 & & & \\
\hline $\begin{array}{l}\text { Api } 5 \text { mg bid } \\
\text { Warfarin INR 2-3 }\end{array}$ & $\begin{array}{c}2.13 / y r \\
3.09 / y r \\
\text { ISTH } \\
\text { P<0.001 }\end{array}$ & $\begin{array}{l}0.33 / \mathrm{yr} \\
0.80 / \mathrm{yr} \\
\mathrm{P}<0.001\end{array}$ & & & $\begin{array}{l}4.07 / \mathrm{yr} \\
6.01 / \mathrm{yr} \\
\mathrm{P}<0.001\end{array}$ & & $\begin{array}{l}18.1 / \mathrm{yr} \\
25.8 / \mathrm{yr} \\
\mathrm{P}<0.001\end{array}$ & & [45] \\
\hline $\begin{array}{l}\text { Dab } 110 \text { mg bid } \\
\text { Dab } 150 \text { mg bid } \\
\text { Warfarin INR 2-3 }\end{array}$ & $\begin{array}{l}2.71 / \mathrm{yr} \\
3.11 / \mathrm{yr} \\
3.36 / \mathrm{yr} \\
\mathrm{P}=0.003\end{array}$ & $\begin{array}{l}0.23 / \mathrm{yr} \\
0.30 / \mathrm{yr} \\
0.74 / \mathrm{yr} \\
\mathrm{P}<0.001\end{array}$ & & & & $\begin{array}{l}13.2 / \mathrm{yr} \\
14.8 / \mathrm{yr} \\
16.4 / \mathrm{yr} \\
\mathrm{P}<0.001\end{array}$ & & & [46] \\
\hline $\begin{array}{l}\text { Dab } 150 \text { mg bid } \\
\text { Warfarin INR 2-3 }\end{array}$ & $\begin{array}{c}1.6 \% \\
6.9 \% \\
P<0.0001\end{array}$ & & & & & $\begin{array}{l}19 \% \\
17 \%\end{array}$ & & & [47] \\
\hline $\begin{array}{l}\text { Dab } 110 \text { mg bid } \\
\text { Warfarin/Aspirin } \\
\text { Dab } 150 \text { mg bid } \\
\text { Warfarin/Aspirin }\end{array}$ & $\begin{array}{c}5.0 \% \\
9.2 \% \\
5.6 \% \\
8.4 \% \\
\mathrm{P}<0.02 \\
\end{array}$ & $\begin{array}{l}0.3 \% \\
1.0 \% \\
0.1 \% \\
1.0 \%\end{array}$ & & & & & & & [48] \\
\hline $\begin{array}{l}\text { Edo } 30 \mathrm{mg} \text { qd } \\
\text { Edo } 60 \mathrm{mg} \text { qd } \\
\text { Warfarin INR 2-3 }\end{array}$ & $\begin{array}{l}1.61 / \mathrm{yr} \\
2.75 / \mathrm{yr} \\
3.43 / \mathrm{yr} \\
\mathrm{P}<0.001 \\
\end{array}$ & $\begin{array}{l}0.26 / \mathrm{yr} \\
0.39 / \mathrm{yr} \\
0.85 / \mathrm{yr} \\
\mathrm{P}<0.001 \\
\end{array}$ & $\begin{array}{l}0.08 / \mathrm{yr} \\
0.15 / \mathrm{yr} \\
0.27 / \mathrm{yr} \\
\mathrm{P}=0.03 \\
\end{array}$ & $\begin{array}{l}6.60 / \mathrm{yr} \\
8.67 / \mathrm{yr} \\
10.2 / \mathrm{yr} \\
\mathrm{P}<0.001\end{array}$ & $\begin{array}{l}7.97 / \mathrm{yr} \\
11.1 / \mathrm{yr} \\
13.0 / \mathrm{yr} \\
\mathrm{P}<0.001 \\
\end{array}$ & $\begin{array}{l}3.52 / \mathrm{yr} \\
4.12 / \mathrm{yr} \\
4.89 / \mathrm{yr} \\
\mathrm{P}=0.002\end{array}$ & & $\begin{array}{l}0.44 / \mathrm{yr} \\
0.70 / \mathrm{yr} \\
1.36 / \mathrm{yr} \\
\\
\mathrm{P}<0.001 \\
\end{array}$ & [49] \\
\hline $\begin{array}{l}\text { Riv } 20 \text { mg qd } \\
\text { Warfarin INR 2-3 }\end{array}$ & $\begin{array}{l}3.6 / \mathrm{yr} \\
3.4 / \mathrm{yr}\end{array}$ & $\begin{array}{l}0.5 / \mathrm{yr} \\
0.7 / \mathrm{yr} \\
\mathrm{P}=0.02\end{array}$ & $\begin{array}{c}0.2 / \mathrm{yr} \\
0.5 / \mathrm{yr} \\
\mathrm{P}=0.003\end{array}$ & $\begin{array}{l}11.8 / \mathrm{yr} \\
11.4 / \mathrm{yr}\end{array}$ & & & & $\begin{array}{c}0.8 \\
1.2 \\
\mathrm{P}=0.007\end{array}$ & [50] \\
\hline $\begin{array}{l}\text { Riv } 20 \text { mg qd } \\
\text { Vit K antag INR 2-3 }\end{array}$ & $\begin{array}{l}0.61 \\
0.80\end{array}$ & $\begin{array}{l}0.20 \\
0.20\end{array}$ & $\begin{array}{l}0.10 \\
0.40\end{array}$ & & & & & $\begin{array}{l}0.20 \\
0.60\end{array}$ & {$[51]$} \\
\hline
\end{tabular}

Table 12: Comparative studies in atrial fibrillation, safety results, bleeding. 


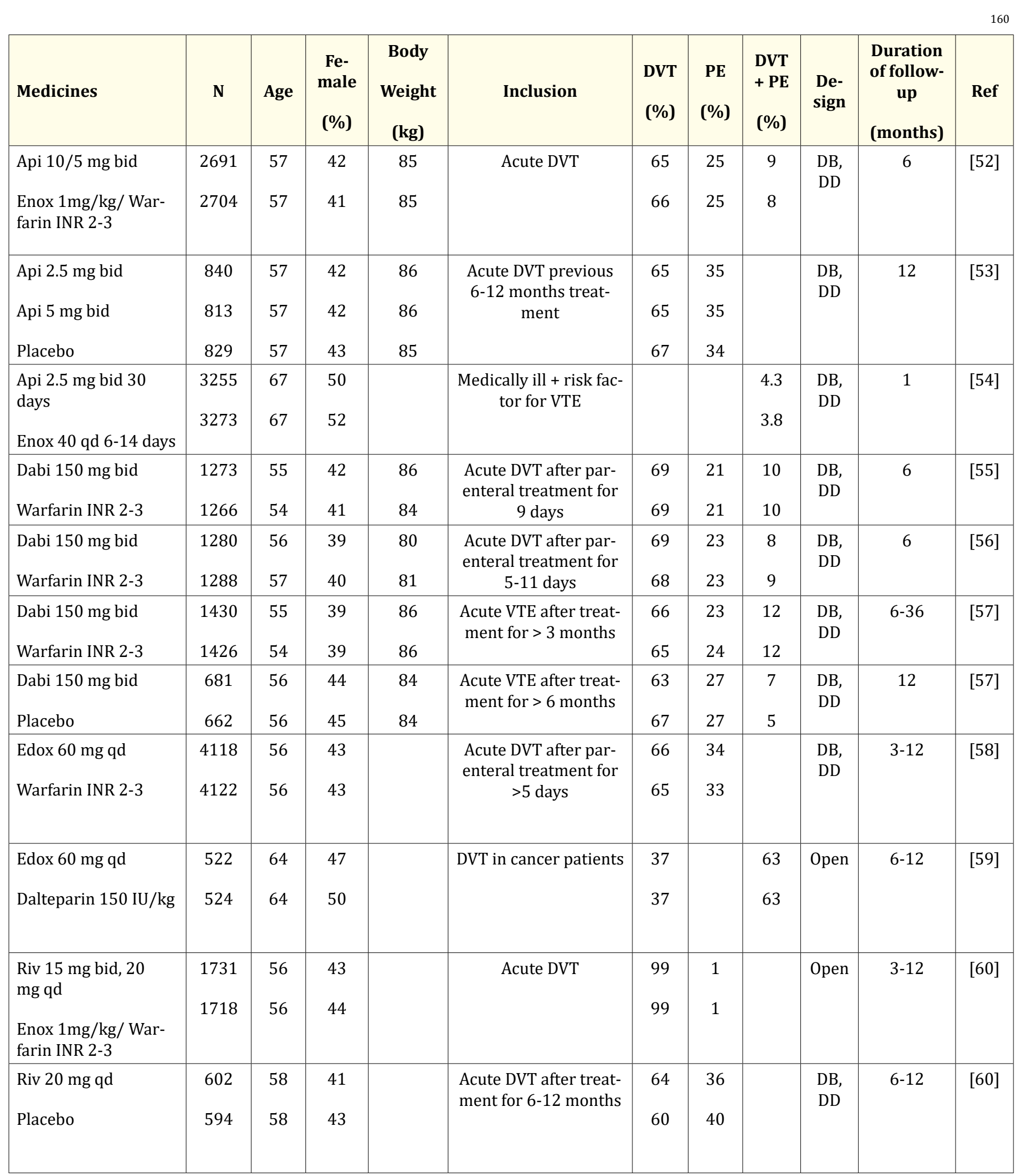




\begin{tabular}{|c|c|c|c|c|c|c|c|c|c|c|c|}
\hline $\begin{array}{l}\text { Riv } 15 \text { mg bid, } 20 \\
\text { mg qd } \\
\text { Enox } 1 \mathrm{mg} / \mathrm{kg} / \text { War- } \\
\text { farin INR } 2-3\end{array}$ & $\begin{array}{l}2419 \\
2413\end{array}$ & $\begin{array}{l}58 \\
58\end{array}$ & $\begin{array}{l}46 \\
48\end{array}$ & & Acute PE & $\begin{array}{l}0 \\
0\end{array}$ & $\begin{array}{l}75 \\
75\end{array}$ & $\begin{array}{l}25 \\
25\end{array}$ & Open & $3-12$ & [61] \\
\hline $\begin{array}{l}\text { Riv } 10 \mathrm{mg} \text { qd } 35 \text { days } \\
\text { Enox } 40 \mathrm{mg} \text { qd } 10 \\
\text { days }\end{array}$ & $\begin{array}{l}4050 \\
4051\end{array}$ & $\begin{array}{l}71 \\
71\end{array}$ & $\begin{array}{l}44 \\
47\end{array}$ & $\begin{array}{l}78 \\
77\end{array}$ & Acutely ill medical & 0 & 0 & 0 & $\begin{array}{l}\text { DB, } \\
\text { DD }\end{array}$ & 35 days & {$[62]$} \\
\hline $\begin{array}{l}\text { Riv } 10 \mathrm{mg} \\
\text { Riv } 20 \mathrm{mg} \\
\text { Aspirin } 100 \mathrm{mg}\end{array}$ & $\begin{array}{l}1127 \\
1107 \\
1131\end{array}$ & $\begin{array}{l}59 \\
58 \\
59\end{array}$ & $\begin{array}{l}45 \\
46 \\
43\end{array}$ & & $\begin{array}{c}\text { Extended treatment } \\
\text { after initial } 6-12 \\
\text { months }\end{array}$ & $\begin{array}{l}50 \\
51 \\
51\end{array}$ & $\begin{array}{l}34 \\
34 \\
32\end{array}$ & $\begin{array}{l}16 \\
14 \\
16\end{array}$ & DB & 12 months & {$[63]$} \\
\hline
\end{tabular}

Table 13: Comparative studies in deep venous thrombosis, baseline data and design.

\begin{tabular}{|c|c|c|c|c|c|c|c|c|c|c|}
\hline Medicines & $\begin{array}{c}\mathrm{C} \mathrm{CR} \\
>50 \\
\mathrm{ml} / \mathrm{min} \\
\\
\%\end{array}$ & $\begin{array}{l}\text { Previ- } \\
\text { ous } \\
\text { VTE } \\
(\%)\end{array}$ & $\begin{array}{c}\text { Unpro- } \\
\text { voked } \\
\text { VTE } \\
(\%)\end{array}$ & 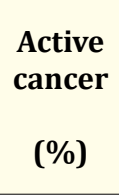 & $\begin{array}{c}\begin{array}{c}\text { Coronary } \\
\text { artery } \\
\text { disease }\end{array} \\
(\%)\end{array}$ & $\begin{array}{l}\text { Diabe- } \\
\text { tes } \\
(\%)\end{array}$ & $\begin{array}{c}\begin{array}{c}\text { Heart } \\
\text { failure }\end{array} \\
(\%)\end{array}$ & $\begin{array}{c}\text { Immo- } \\
\text { bilised } \\
(\%)\end{array}$ & $\begin{array}{l}\text { Primary end- } \\
\text { point }\end{array}$ & Ref \\
\hline $\begin{array}{l}\text { Api 10/5 mg bid } \\
\text { Enox } 1 \mathrm{mg} / \mathrm{kg} / \\
\text { Warfarin INR 2-3 }\end{array}$ & $\begin{array}{l}84 \\
85\end{array}$ & $\begin{array}{l}17 \\
15\end{array}$ & $\begin{array}{l}90 \\
90\end{array}$ & $\begin{array}{l}2.5 \\
2.8\end{array}$ & & & & & $\begin{array}{l}\text { VTE-RS or } \\
\text { VTE-D }\end{array}$ & [52] \\
\hline $\begin{array}{l}\text { Api } 2.5 \mathrm{mg} \text { bid } \\
\text { Api } 5 \mathrm{mg} \text { bid } \\
\text { Placebo }\end{array}$ & $\begin{array}{l}91 \\
92 \\
91\end{array}$ & $\begin{array}{l}12 \\
15 \\
12\end{array}$ & $\begin{array}{l}93 \\
91 \\
91\end{array}$ & $\begin{array}{l}1.8 \\
1.1 \\
2.2\end{array}$ & & $\begin{array}{l}12 \\
10 \\
11\end{array}$ & & $\begin{array}{l}2.3 \\
3.6 \\
2.7\end{array}$ & $\begin{array}{l}\text { VTE-RS or } \\
\text { death }\end{array}$ & [53] \\
\hline $\begin{array}{l}\text { Api } 2.5 \mathrm{mg} \text { bid } \\
30 \mathrm{~d} \\
\text { Enox } 40 \mathrm{qd} 6-14 \mathrm{~d}\end{array}$ & & $\begin{array}{l}4.3 \\
3.8\end{array}$ & & $\begin{array}{l}3.5 \\
3.0\end{array}$ & & & $\begin{array}{l}39 \\
38\end{array}$ & & $\begin{array}{l}\text { VTE-D or PE } \\
\text { or DVT-S or } \\
\text { DVT-P }\end{array}$ & [54] \\
\hline $\begin{array}{l}\text { Dabi } 150 \mathrm{mg} \text { bid } \\
\text { Warfarin INR 2-3 }\end{array}$ & & $\begin{array}{l}26 \\
25\end{array}$ & & $\begin{array}{l}5.0 \\
4.5 \\
\end{array}$ & & & & & $\begin{array}{l}\text { VTE-RS or } \\
\text { VTE-D }\end{array}$ & [55] \\
\hline $\begin{array}{l}\text { Dabi } 150 \mathrm{mg} \text { bid } \\
\text { Warfarin INR 2-3 }\end{array}$ & & $\begin{array}{l}19 \\
16\end{array}$ & & $\begin{array}{l}3.9 \\
3.9\end{array}$ & & & & & $\begin{array}{l}\text { VTE-RS or } \\
\text { VTE-D }\end{array}$ & [56] \\
\hline $\begin{array}{l}\text { Dabi } 150 \mathrm{mg} \text { bid } \\
\text { Warfarin INR 2-3 }\end{array}$ & & & & $\begin{array}{l}4.2 \\
4.1\end{array}$ & $\begin{array}{l}8.4 \\
6.1 \\
\end{array}$ & $\begin{array}{r}10.5 \\
7.6 \\
\end{array}$ & & $\begin{array}{l}6.6 \\
7.3\end{array}$ & $\begin{array}{l}\text { VTE-RS or } \\
\text { VTE-D }\end{array}$ & [57] \\
\hline $\begin{array}{l}\text { Dabi } 150 \mathrm{mg} \text { bid } \\
\text { Placebo }\end{array}$ & & & & & $\begin{array}{l}6.3 \\
5.7 \\
\end{array}$ & $\begin{array}{l}8.4 \\
7.6 \\
\end{array}$ & & $\begin{array}{l}7.8 \\
5.4 \\
\end{array}$ & $\begin{array}{l}\text { VTE-RS or } \\
\text { VTE-D }\end{array}$ & [57] \\
\hline $\begin{array}{l}\text { Edox } 60 \text { mg qd } \\
\text { Warfarin INR 2-3 }\end{array}$ & & $\begin{array}{l}19 \\
18\end{array}$ & $\begin{array}{l}66 \\
65\end{array}$ & $\begin{array}{l}9.2 \\
9.5\end{array}$ & & & & & $\begin{array}{l}\text { VTE-RS or } \\
\text { VTE-D }\end{array}$ & [58] \\
\hline
\end{tabular}




\begin{tabular}{|c|c|c|c|c|c|c|c|c|}
\hline $\begin{array}{l}\text { Edox } 60 \text { mg qd } \\
\text { Dalteparin } 150 \\
\text { IU/kg }\end{array}$ & $\begin{array}{l}93 \\
93\end{array}$ & $\begin{array}{c}9 \\
12\end{array}$ & & $\begin{array}{l}98 \\
98\end{array}$ & & & $\begin{array}{l}\text { VTE or bleed- } \\
\text { ing }\end{array}$ & [59] \\
\hline $\begin{array}{l}\text { Riv } 15 \text { mg bid, } 20 \\
\text { mg qd } \\
\text { Enox } 1 \mathrm{mg} / \mathrm{kg} / \\
\text { Warfarin INR 2-3 }\end{array}$ & & & $\begin{array}{l}61 \\
63\end{array}$ & $\begin{array}{l}6.8 \\
5.2\end{array}$ & & $\begin{array}{l}15 \\
15\end{array}$ & VTE-RS & [60] \\
\hline $\begin{array}{l}\text { Riv } 20 \text { mg qd } \\
\text { Placebo }\end{array}$ & & & $\begin{array}{l}73 \\
74 \\
\end{array}$ & $\begin{array}{l}4.7 \\
4.4 \\
\end{array}$ & & $\begin{array}{l}15 \\
13 \\
\end{array}$ & VTE-RS & [60] \\
\hline $\begin{array}{l}\text { Riv } 15 \text { mg bid, } 20 \\
\text { mg qd } \\
\text { Enox } 1 \mathrm{mg} / \mathrm{kg} / \\
\text { Warfarin INR 2-3 }\end{array}$ & $\begin{array}{l}91 \\
92\end{array}$ & $\begin{array}{l}19 \\
20\end{array}$ & $\begin{array}{l}65 \\
64\end{array}$ & $\begin{array}{l}4.7 \\
4.5\end{array}$ & & $\begin{array}{l}16 \\
16\end{array}$ & VTE-RS & [61] \\
\hline $\begin{array}{l}\text { Riv } 10 \text { mg qd } 35 \\
\text { days } \\
\text { Enox } 40 \text { mg qd } 10 \\
\text { days }\end{array}$ & & & & $\begin{array}{l}7.3 \\
7.3\end{array}$ & $\begin{array}{l}32 \\
32\end{array}$ & & VTE & [62] \\
\hline $\begin{array}{l}\text { Riv } 10 \mathrm{mg} \\
\text { Riv } 20 \mathrm{mg} \\
\text { Aspirin } 100 \mathrm{mg}\end{array}$ & & & & $\begin{array}{l}2.4 \\
2.3 \\
3.3\end{array}$ & & & VTE-RS & [63] \\
\hline
\end{tabular}

Table 14: Comparative studies in deep venous thrombosis, baseline data and design.

UA: Instable Angina

VTE-RS: VTE recurrent symptomatic thromboembolism

VTE-D: VTE related death.

\begin{tabular}{|c|c|c|c|c|c|c|c|c|c|c|c|}
\hline Medicines & $\begin{array}{l}\text { VTE-RS or } \\
\text { VTE-D } \\
\text { (\%) }\end{array}$ & $\begin{array}{l}\text { VTE- } \\
\text { RS or } \\
\text { death } \\
\text { (\%) }\end{array}$ & $\begin{array}{l}\text { VTE-D } \\
\text { or PE } \\
\text { or DVT- } \\
\text { S or } \\
\text { DVT-P }\end{array}$ & $\begin{array}{c}\text { CV-D, } \\
\text { MI, } \\
\text { stroke }\end{array}$ & $\begin{array}{l}\text { PE- } \\
\text { NF } \\
(\%)\end{array}$ & $\begin{array}{l}\text { PE-F } \\
\text { (\%) }\end{array}$ & $\begin{array}{c}\text { VTE-S } \\
\text { (\%) }\end{array}$ & $\begin{array}{l}\text { DVT- } \\
\text { P(\%) }\end{array}$ & $\begin{array}{c}\text { Death } \\
\text { (\%) }\end{array}$ & $\begin{array}{c}\text { VTE-D } \\
\text { (\%) }\end{array}$ & Ref \\
\hline $\begin{array}{l}\text { Api } 10 / 5 \mathrm{mg} \text { bid } \\
\text { Enox } 1 \mathrm{mg} / \mathrm{kg} / \text { Warfarin } \\
\text { INR } 2-3\end{array}$ & $\begin{array}{c}2.3 \\
2.7 \\
\text { NI: } p<0.001\end{array}$ & & & & $\begin{array}{l}1.0 \\
0.9\end{array}$ & $\begin{array}{c}<0.1 \\
0.1\end{array}$ & & & $\begin{array}{l}1.5 \\
1.9\end{array}$ & & [52] \\
\hline $\begin{array}{l}\text { Api } 2.5 \mathrm{mg} \text { bid } \\
\text { Api } 5 \mathrm{mg} \text { bid } \\
\text { Placebo }\end{array}$ & $\begin{array}{c}1.7 \\
1.7 \\
8.8 \\
P<0.001\end{array}$ & $\begin{array}{c}3.8 \\
4.2 \\
11.6 \\
P<0.001\end{array}$ & & $\begin{array}{l}0.5 \\
0.6 \\
1.3\end{array}$ & $\begin{array}{l}1.0 \\
0.5 \\
1.8\end{array}$ & $\begin{array}{l}0 \\
0 \\
0\end{array}$ & & & & & [53] \\
\hline
\end{tabular}


163

\begin{tabular}{|c|c|c|c|c|c|c|c|c|c|c|}
\hline $\begin{array}{l}\text { Api } 2.5 \mathrm{mg} \text { bid } 30 \mathrm{~d} \\
\text { Enox } 40 \mathrm{qd} 6-14 \mathrm{~d}\end{array}$ & & $\begin{array}{l}2.71 \\
3.06 \\
\end{array}$ & & $\begin{array}{l}0.22 \\
0.24\end{array}$ & $\begin{array}{l}0 \\
0\end{array}$ & $\begin{array}{l}0.15 \\
0.49 \\
\end{array}$ & $\begin{array}{l}2.40 \\
2.50\end{array}$ & $\begin{array}{l}4.1 \\
4.1 \\
\end{array}$ & $\begin{array}{l}0.06 \\
0.09\end{array}$ & [54] \\
\hline $\begin{array}{l}\text { Dabi } 150 \text { mg bid } \\
\text { Warfarin INR 2-3 }\end{array}$ & $\begin{array}{l}2.4 \\
2.1 \\
\end{array}$ & & & $\begin{array}{l}1.0 \\
0.6 \\
\end{array}$ & & $\begin{array}{l}1.3 \\
1.4 \\
\end{array}$ & & $\begin{array}{l}1.6 \\
1.7 \\
\end{array}$ & $\begin{array}{l}0.1 \\
0.2 \\
\end{array}$ & [55] \\
\hline $\begin{array}{l}\text { Dabi } 150 \text { mg bid } \\
\text { Warfarin INR 2-3 }\end{array}$ & $\begin{array}{l}2.3 \\
2.3\end{array}$ & & & $\begin{array}{l}0.5 \\
1.0\end{array}$ & & $\begin{array}{l}2.0 \\
1.3\end{array}$ & & $\begin{array}{l}2.0 \\
1.9\end{array}$ & $\begin{array}{c}0.2 \\
0 \\
\end{array}$ & [56] \\
\hline $\begin{array}{l}\text { Dabi } 150 \text { mg bid } \\
\text { Warfarin INR 2-3 }\end{array}$ & $\begin{array}{c}1.8 \\
1.3 \\
P<0.01 \\
\end{array}$ & & & $\begin{array}{l}0.7 \\
0.4\end{array}$ & & $\begin{array}{l}1.2 \\
0.0\end{array}$ & & $\begin{array}{l}1.2 \\
1.3\end{array}$ & $\begin{array}{l}0.1 \\
0.1\end{array}$ & [57] \\
\hline $\begin{array}{l}\text { Dabi } 150 \mathrm{mg} \text { bid } \\
\text { Placebo }\end{array}$ & $\begin{array}{c}0.4 \\
5.6 \\
<<0.001 \\
\end{array}$ & & & $\begin{array}{l}0.1 \\
2.1\end{array}$ & & $\begin{array}{l}0.3 \\
3.3 \\
\text { NS } \\
\end{array}$ & & & & [57] \\
\hline $\begin{array}{l}\text { Edox } 60 \mathrm{mg} \text { qd } \\
\text { Warfarin INR 2-3 }\end{array}$ & $\begin{array}{c}3.2 \\
3.5 \\
\text { NI: } \mathrm{p}<0.001\end{array}$ & & & $\begin{array}{l}1.2 \\
1.4\end{array}$ & $\begin{array}{l}0.1 \\
0.1\end{array}$ & $\begin{array}{l}1.4 \\
1.5\end{array}$ & & $\begin{array}{l}0.5 \\
0.5\end{array}$ & $\begin{array}{l}0.5 \\
0.5\end{array}$ & [C58] \\
\hline $\begin{array}{l}\text { Edox } 60 \text { mg qd } \\
\text { Dalteparin } 150 \mathrm{IU} / \mathrm{kg}\end{array}$ & $\begin{array}{r}7.9 \\
11.3 \\
\end{array}$ & & & $\begin{array}{l}5.2 \\
5.3 \\
\end{array}$ & & & & $\begin{array}{l}40 \\
37 \\
\end{array}$ & $\begin{array}{l}0.6 \\
0.6 \\
\end{array}$ & [59] \\
\hline $\begin{array}{l}\text { Riv } 15 \mathrm{mg} \text { bid, } 20 \mathrm{mg} \text { qd } \\
\text { Enox } 1 \mathrm{mg} / \mathrm{kg} / \text { Warfarin } \\
\text { INR } 2-3\end{array}$ & & & & $\begin{array}{l}1.2 \\
1.1\end{array}$ & $\begin{array}{c}0.1 \\
0\end{array}$ & $\begin{array}{c}2.1 \\
3.0 \\
\text { NI: } \\
\mathrm{P}<0.001 \\
\end{array}$ & & $\begin{array}{l}2.2 \\
2.9\end{array}$ & & [60] \\
\hline $\begin{array}{l}\text { Riv } 20 \mathrm{mg} \mathrm{qd} \\
\text { Placebo }\end{array}$ & & & & $\begin{array}{l}0.3 \\
2.8\end{array}$ & $\begin{array}{c}0 \\
0.2\end{array}$ & $\begin{array}{c}1.3 \\
7.1 \\
\mathrm{P}<0.001 \\
\end{array}$ & & $\begin{array}{l}0.2 \\
0.3\end{array}$ & & {$[60]$} \\
\hline $\begin{array}{l}\text { Riv } 15 \mathrm{mg} \text { bid, } 20 \mathrm{mg} \text { qd } \\
\text { Enox } 1 \mathrm{mg} / \mathrm{kg} / \text { Warfarin } \\
\text { INR } 2-3\end{array}$ & & & & $\begin{array}{l}0.9 \\
0.7\end{array}$ & $\begin{array}{l}0.1 \\
<0.1\end{array}$ & $\begin{array}{c}2.1 \\
1.8 \\
\mathrm{NI} \\
\mathrm{p}=0.003 \\
\end{array}$ & & $\begin{array}{l}2.4 \\
2.1\end{array}$ & & [61] \\
\hline $\begin{array}{l}\text { Riv } 10 \mathrm{mg} \text { qd } 35 \text { days } \\
\text { Enox } 40 \mathrm{mg} \text { qd } 10 \text { days }\end{array}$ & & & $\begin{array}{l}1.8 \\
1.6\end{array}$ & $\begin{array}{l}0.3 \\
0.5\end{array}$ & & $\begin{array}{c}4.4 \\
5.7 \\
\mathrm{p}=0.02 \\
\end{array}$ & $\begin{array}{l}3.5 \\
4.4\end{array}$ & $\begin{array}{l}5.1 \\
4.8\end{array}$ & $\begin{array}{l}0.6 \\
1.0\end{array}$ & {$[62]$} \\
\hline $\begin{array}{l}\text { Riv } 10 \mathrm{mg} \\
\text { Riv } 20 \mathrm{mg} \\
\text { Aspirin } 100 \mathrm{mg}\end{array}$ & $\begin{array}{c}1.2 \\
1.5 \\
4.4 \\
\mathrm{P}<0.001 \\
\end{array}$ & & $\begin{array}{l}1.6 \\
1.7 \\
5.0\end{array}$ & $\begin{array}{l}0.4 \\
0.5 \\
1.7\end{array}$ & & & & $\begin{array}{l}0.2 \\
0.7 \\
0.6\end{array}$ & & [63] \\
\hline
\end{tabular}

Table 15: Comparative studies in deep venous thrombosis, results. 
164



Table 16: Comparative studies in deep venous thrombosis, safety results. 


\begin{tabular}{|c|c|c|c|c|c|c|c|c|c|}
\hline Medicines & $\begin{array}{l}\text { Major } \\
\text { bleed- } \\
\text { ing }\end{array}$ & $\begin{array}{l}\text { Intra- } \\
\text { cranial } \\
\text { bleed- } \\
\text { ing }\end{array}$ & $\begin{array}{c}\text { Fatal } \\
\text { bleeding }\end{array}$ & $\begin{array}{c}\text { Clinically } \\
\text { relevant } \\
\text { non- } \\
\text { major }\end{array}$ & $\begin{array}{l}\text { Major or } \\
\text { Clinically } \\
\text { relevant } \\
\text { non-major }\end{array}$ & $\begin{array}{c}\text { Minor } \\
\text { bleeding }\end{array}$ & $\begin{array}{l}\text { Any } \\
\text { bleed- } \\
\text { ing }\end{array}$ & $\begin{array}{l}\text { Bleeding } \\
\text { in criti- } \\
\text { cal organ }\end{array}$ & Ref \\
\hline Api $10 / 5 \mathrm{mg}$ bid & 0.6 & 0.1 & $<0.1$ & 3.8 & 4.3 & & 16 & & {$[52]$} \\
\hline $\begin{array}{l}\text { Enox } 1 \mathrm{mg} / \mathrm{kg} / \text { Warfarin } \\
\text { INR } 2-3\end{array}$ & $\begin{array}{c}1.8 \\
P<0.001 \\
\end{array}$ & 0.2 & 0.1 & 8.0 & $\begin{array}{c}9.7 \\
P<0.001 \\
\end{array}$ & & 26 & & \\
\hline Api $2.5 \mathrm{mg}$ bid & 0.2 & & 0 & 3.0 & 3.2 & & & 0.2 & {$[53]$} \\
\hline Api $5 \mathrm{mg}$ bid & 0.1 & & 0 & 4.2 & 4.3 & & & 0 & \\
\hline Placebo & 0.5 & & 0 & 2.3 & 2.7 & & & 0.2 & \\
\hline Api $2.5 \mathrm{mg}$ bid $30 \mathrm{~d}$ & 0.47 & 0 & 0 & 2.2 & 2.7 & & 7.7 & $<0.1$ & {$[54]$} \\
\hline Enox $40 \mathrm{qd}$ 6-14 d & $\begin{array}{c}0.19 \\
P=0.04\end{array}$ & 0.1 & 0.1 & 1.9 & 2.1 & & 6.8 & 0 & \\
\hline Dabi 150 mg bid & 1.6 & 0 & 0.1 & 4.0 & 5.6 & & 16.1 & 0.1 & {$[55]$} \\
\hline Warfarin INR 2-3 & 1.9 & 0.3 & 0.1 & 6.9 & $\begin{array}{c}8.8 \\
\mathrm{P}=0.02 \\
\end{array}$ & & 21.9 & 0.7 & \\
\hline Dabi 150 mg bid & 1.2 & 0.2 & 0 & & 5.0 & & & 0.5 & {$[56]$} \\
\hline Warfarin INR 2-3 & 1.7 & 0.2 & 0.1 & & $\begin{array}{c}7.9 \\
\text { P not stated }\end{array}$ & & & 0.3 & \\
\hline Dabi $150 \mathrm{mg}$ bid & 0.9 & & 0 & & 5.6 & & 19.4 & 0.5 & {$[57]$} \\
\hline Warfarin INR 2-3 & 1.8 & & 0.1 & & $\begin{array}{c}10.2 \\
P<0.001\end{array}$ & & $\begin{array}{c}26.2 \\
P<0.001\end{array}$ & 0.9 & \\
\hline $\begin{array}{l}\text { Dabi } 150 \text { mg bid } \\
\text { Placebo }\end{array}$ & $\begin{array}{c}0.3 \\
0\end{array}$ & & & & $\begin{array}{c}5.3 \\
1.8 \\
P<0.001\end{array}$ & & $\begin{array}{c}10.5 \\
5.9 \\
P<0.001\end{array}$ & & [57] \\
\hline $\begin{array}{l}\text { Edox } 60 \text { mg qd } \\
\text { Warfarin INR 2-3 }\end{array}$ & $\begin{array}{l}1.4 \\
1.6\end{array}$ & $\begin{array}{l}0.1 \\
0.4\end{array}$ & $\begin{array}{c}<0.1 \\
0.2\end{array}$ & $\begin{array}{c}7.2 \\
8.9 \\
P=0.004\end{array}$ & & $\begin{array}{c}21.7 \\
25.6 \\
P<0.001\end{array}$ & & $\begin{array}{l}0.3 \\
0.6\end{array}$ & [C58] \\
\hline $\begin{array}{l}\text { Edox } 60 \text { mg qd } \\
\text { Dalteparin } 150 \mathrm{IU} / \mathrm{kg}\end{array}$ & $\begin{array}{l}6.9 \\
4.0 \\
\end{array}$ & & & $\begin{array}{l}15 \\
11 \\
\end{array}$ & $\begin{array}{l}19 \\
14\end{array}$ & & & & [59] \\
\hline $\begin{array}{l}\text { Riv } 15 \mathrm{mg} \text { bid, } 20 \text { mg qd } \\
\text { Enox } 1 \mathrm{mg} / \mathrm{kg} / \text { Warfarin } \\
\text { INR } 2-3\end{array}$ & $\begin{array}{l}0.8 \\
1.2\end{array}$ & & $\begin{array}{l}0.1 \\
0.3\end{array}$ & $\begin{array}{l}7.3 \\
7.0\end{array}$ & & & & $\begin{array}{l}0.2 \\
0.2\end{array}$ & {$[60]$} \\
\hline $\begin{array}{l}\text { Riv } 20 \text { mg qd } \\
\text { Placebo }\end{array}$ & $\begin{array}{c}0.7 \\
0 \\
\end{array}$ & & $\begin{array}{l}0 \\
0 \\
\end{array}$ & $\begin{array}{l}5.4 \\
1.2 \\
\end{array}$ & & & & $\begin{array}{l}0 \\
0 \\
\end{array}$ & {$[60]$} \\
\hline $\begin{array}{l}\text { Riv } 15 \mathrm{mg} \text { bid, } 20 \mathrm{mg} \text { qd } \\
\text { Enox } 1 \mathrm{mg} / \mathrm{kg} / \text { Warfarin } \\
\text { INR } 2-3\end{array}$ & $\begin{array}{c}1.1 \\
2.2 \\
P=0.003\end{array}$ & $\begin{array}{l}0.1 \\
0.1\end{array}$ & $\begin{array}{l}0.1 \\
0.1\end{array}$ & $\begin{array}{l}9.5 \\
9.8\end{array}$ & & & & $\begin{array}{l}0.3 \\
1.1\end{array}$ & {$[61]$} \\
\hline
\end{tabular}




\begin{tabular}{|c|c|c|c|c|c|c|c|}
\hline $\begin{array}{l}\text { Riv } 10 \text { mg qd } 35 \text { days } \\
\text { Enox } 40 \text { mg qd } 10 \text { days }\end{array}$ & $\begin{array}{c}1.2 \\
0.4 \\
\mathrm{P}<0.001 \\
\end{array}$ & & $\begin{array}{l}0.2 \\
<0.1\end{array}$ & $\begin{array}{c}2.8 \\
1.2 \\
\mathrm{P}<0.001 \\
\end{array}$ & & $\begin{array}{l}0.2 \\
0.1\end{array}$ & [62] \\
\hline Riv $10 \mathrm{mg}$ & 0.4 & 0.1 & 0 & 2.4 & 12 & & [63] \\
\hline Riv $20 \mathrm{mg}$ & 0.5 & 0.3 & 0.1 & 3.3 & 15 & & \\
\hline Aspirin $100 \mathrm{mg}$ & 0.3 & 0.2 & 0.1 & 2.0 & 11 & & \\
\hline
\end{tabular}

Table 17: Comparative studies in deep venous thrombosis, safety results, bleeding.

\begin{tabular}{|c|c|c|c|c|c|c|c|c|c|c|c|}
\hline Medicines & $\mathbf{N}$ & Age & $\begin{array}{c}\text { Female } \\
\text { (\%) }\end{array}$ & $\begin{array}{l}\text { De- } \\
\text { sign }\end{array}$ & $\begin{array}{c}\text { Risk } \\
\text { factors }\end{array}$ & & & & & $\begin{array}{c}\text { Duration } \\
\text { of follow- } \\
\text { up }\end{array}$ & Ref \\
\hline & & & & & $\begin{array}{c}\text { Diabe- } \\
\text { tes } \\
\% \\
\%\end{array}$ & $\begin{array}{c}\text { Previous } \\
\text { MI } \\
\text { \% }\end{array}$ & $\begin{array}{c}\text { CV dis- } \\
\text { ease } \\
\% \\
\%\end{array}$ & $\begin{array}{c}\text { LVHF } \\
\quad \%\end{array}$ & $\begin{array}{c}\text { Peripheral vas- } \\
\text { cular disease } \\
\% \\
\end{array}$ & & \\
\hline Api $5 \mathrm{mg}$ bid & 3705 & 67 & 33 & DB & 49 & 25 & 10 & 28 & 18 & 1.25 years & [64] \\
\hline Placebo & 3687 & 67 & 32 & & 47 & 28 & 10 & 29 & 18 & & \\
\hline Riva $2.5 \mathrm{mg}$ bid & 5174 & 62 & 35 & DB & 32 & 26 & & & & $\begin{array}{c}13-31 \\
\text { months }\end{array}$ & [65] \\
\hline Riva $5 \mathrm{mg}$ bid & 5176 & 62 & 36 & & 32 & 27 & & & & & \\
\hline Placebo & 5176 & 62 & 35 & & 32 & 27 & & & & & \\
\hline Riva $2.5 \mathrm{mg}$ bid & 1519 & 62 & 25 & DB & 29 & 21 & & & & 390 days & [66] \\
\hline Aspirin $100 \mathrm{mg} \mathrm{qd}$ & 1518 & 63 & 25 & & 30 & 23 & & & & & \\
\hline
\end{tabular}

Table 18: Comparative studies in acute coronary syndromes, baseline data and design.

\begin{tabular}{|c|c|c|c|c|c|c|c|c|c|c|c|}
\hline Medicines & $\begin{array}{c}\text { Type } \\
\text { of ACS }\end{array}$ & & & $\begin{array}{l}\text { Time from } \\
\text { event to } \\
\text { randomi- } \\
\text { sation } \\
\text { (days) }\end{array}$ & $\begin{array}{l}\text { Medica- } \\
\text { tion }\end{array}$ & & & & & $\begin{array}{l}\text { Primary } \\
\text { Endpoint }\end{array}$ & Ref \\
\hline & STEMI & $\begin{array}{c}\mathrm{N}- \\
\text { STEMI }\end{array}$ & UA & & $\begin{array}{c}\text { ACE/ } \\
\text { A2A }\end{array}$ & $\begin{array}{c}\text { Beta } \\
\text { blocker }\end{array}$ & Statin & $\begin{array}{l}\text { Parenteral } \\
\text { Antithrom- } \\
\text { botic agents }\end{array}$ & PPI & & \\
\hline Api 5 mg bid or & 40 & 41 & 18 & 6.0 & 80 & 77 & 83 & & 24 & $\begin{array}{l}\text { CV-D, MI, } \\
\text { I-S }\end{array}$ & [64] \\
\hline Placebo or & 39 & 42 & 18 & 6.0 & 79 & 76 & 84 & 81 & 25 & & \\
\hline & 50 & 26 & 24 & & 39 & 66 & 83 & & & $\begin{array}{l}\text { CV-D, MI, } \\
\text { Stroke }\end{array}$ & [65] \\
\hline Riva $5 \mathrm{mg}$ bid & 50 & 26 & 24 & & 38 & 66 & 84 & & & & \\
\hline Placebo & 51 & 26 & 24 & & 39 & 67 & 84 & & & & \\
\hline Riva $2.5 \mathrm{mg}$ bid & 49 & 40 & 11 & 5.1 & 62 & 64 & 68 & & & $\begin{array}{l}\text { TIMI } \\
\text { hleeding }\end{array}$ & [66] \\
\hline Aspirin $100 \mathrm{mg} \mathrm{qd}$ & 49 & 40 & 11 & 5.1 & 63 & 65 & 70 & & & & \\
\hline
\end{tabular}

Table 19: Comparative studies in acute coronary syndromes, baseline data and design. 


\begin{tabular}{|c|c|c|c|c|c|c|c|c|c|}
\hline Medicines & $\begin{array}{c}\text { Composite } \\
\text { (\%) }\end{array}$ & & & $\begin{array}{c}\text { Death } \\
\text { (\%) }\end{array}$ & $\begin{array}{l}\text { CV-D } \\
(\%)\end{array}$ & $\begin{array}{l}\text { MI } \\
\text { (\%) }\end{array}$ & $\begin{array}{l}\text { I-S } \\
(\%)\end{array}$ & $\begin{array}{c}\text { Stent } \\
\text { thrombosis } \\
\text { (\%) }\end{array}$ & Ref \\
\hline & CV-D, MI, I-S & $\begin{array}{c}\text { CV-D, } \\
\text { MI, I-S } \\
\text { UA }\end{array}$ & $\begin{array}{l}\text { CV-D, MI, } \\
\text { stroke }\end{array}$ & & & & & & \\
\hline $\begin{array}{l}\text { Api } 5 \text { mg bid } \\
\text { or } \\
\text { Placebo or }\end{array}$ & $\begin{array}{l}7.5 \\
7.9\end{array}$ & $\begin{array}{c}9.5 \\
10.0\end{array}$ & & $\begin{array}{l}4.2 \\
3.9\end{array}$ & $\begin{array}{l}4.8 \\
5.0\end{array}$ & $\begin{array}{l}8.6 \\
9.2\end{array}$ & $\begin{array}{l}0.6 \\
1.6\end{array}$ & $\begin{array}{l}0.9 \\
1.3\end{array}$ & [64] \\
\hline $\begin{array}{l}\text { Riva } 2.5 \mathrm{mg} \\
\text { bid } \\
\text { Riva } 5 \mathrm{mg} \text { bid } \\
\text { Placebo }\end{array}$ & & & $\begin{array}{c}9.1 \\
8.8 \\
10.7 \\
P=0.02(2.5 \mathrm{mg} \\
\text { vs placebo })\end{array}$ & $\begin{array}{c}2.9 \\
4.4 \\
4.5 \\
\mathrm{P}=0.002(2.5 \\
\mathrm{mg} \text { vs placebo })\end{array}$ & $\begin{array}{c}2.7 \\
4.0 \\
4.1 \\
\mathrm{P}=0.002(2.5 \\
\text { mg vs placebo) }\end{array}$ & & & $\begin{array}{l}2.2 \\
2.3 \\
2.9\end{array}$ & [65] \\
\hline $\begin{array}{l}\text { Riva } 2.5 \text { mg } \\
\text { bid } \\
\text { Aspirin } 100 \\
\text { mg qd }\end{array}$ & $\begin{array}{c}5 \\
5 \\
\\
\text { (including stent } \\
\text { thrombosis) }\end{array}$ & & & & $\begin{array}{l}0.6 \\
0.6\end{array}$ & $\begin{array}{l}3.6 \\
3.3\end{array}$ & & $\begin{array}{l}0.6 \\
0.8\end{array}$ & [66] \\
\hline
\end{tabular}

Table 20: Comparative studies in acute coronary syndromes, results.

\begin{tabular}{|c|c|c|c|c|c|c|c|c|c|c|}
\hline Medicines & $\begin{array}{c}\text { Major } \\
\text { bleed- } \\
\text { ing } \\
\text { TIMI } \\
(\%)\end{array}$ & $\begin{array}{c}\begin{array}{c}\text { Major or } \\
\text { minor } \\
\text { bleeding }\end{array} \\
\text { TIMI (\%) }\end{array}$ & $\begin{array}{c}\text { Major } \\
\text { bleed- } \\
\text { ing } \\
\text { ISTH } \\
\\
(\%)\end{array}$ & $\begin{array}{c}\text { Major or } \\
\text { clinically } \\
\text { relevant } \\
\text { non-major } \\
\text { bleeding } \\
\text { (\%) }\end{array}$ & $\begin{array}{c}\begin{array}{c}\text { Severe } \\
\text { bleeding }\end{array} \\
\text { GUST0 } \\
\text { (\%) }\end{array}$ & $\begin{array}{c}\text { Severe or } \\
\text { moderate } \\
\text { bleeding } \\
\text { GUSTO } \\
\%\end{array}$ & $\begin{array}{c}\text { Fatal } \\
\text { bleeding }\end{array}$ & $\begin{array}{c}\text { Intracrani- } \\
\text { al bleeding } \\
\text { (\%) }\end{array}$ & $\begin{array}{c}\text { Any } \\
\text { bleed- } \\
\text { ing (\%) }\end{array}$ & Ref \\
\hline $\begin{array}{l}\text { Api } 5 \mathrm{mg} \text { bid or } \\
\text { Placebo or }\end{array}$ & $\begin{array}{c}1.3 \\
0.5 \\
\mathrm{P}=0.001\end{array}$ & $\begin{array}{c}2.2 \\
0.8 \\
\mathrm{P}<0.001\end{array}$ & $\begin{array}{c}2.7 \\
1.1 \\
\mathrm{P}<0.001 \\
\end{array}$ & $\begin{array}{c}3.2 \\
1.2 \\
\mathrm{P}<0.001\end{array}$ & $\begin{array}{c}1.8 \\
0.6 \\
\mathrm{P}<0.001\end{array}$ & $\begin{array}{c}2.3 \\
0.7 \\
P<0.001\end{array}$ & $\begin{array}{c}0.1 \\
0\end{array}$ & $\begin{array}{c}0.6 \\
0.2 \\
\mathrm{P}=0.03\end{array}$ & $\begin{array}{c}18.5 \\
14.4 \\
P<0.001 \\
\end{array}$ & [64] \\
\hline $\begin{array}{l}\text { Riva } 2.5 \mathrm{mg} \text { bid } \\
\text { Riva } 5 \mathrm{mg} \text { bid } \\
\text { Placebo }\end{array}$ & $\begin{array}{l}1.8 \\
2.4 \\
0.6\end{array}$ & & & & & & $\begin{array}{c}0.1 \\
0.4 \\
0.2 \\
\mathrm{P}=0.04 \text { for } \\
2.5 \mathrm{mg} \text { vs } \\
5 \mathrm{mg} \text { Riva }\end{array}$ & $\begin{array}{c}0.4 \\
0.7 \\
0.2 \\
\mathrm{P}=0.009\end{array}$ & & [65] \\
\hline $\begin{array}{l}\text { Riva } 2.5 \mathrm{mg} \text { bid } \\
\text { Aspirin } 100 \\
\mathrm{mg} \mathrm{qd}\end{array}$ & $\begin{array}{l}0.6 \\
0.5\end{array}$ & & $\begin{array}{l}2.0 \\
1.1\end{array}$ & & $\begin{array}{l}0.2 \\
0.1\end{array}$ & & $\begin{array}{c}0.1 \\
0\end{array}$ & & & [66] \\
\hline
\end{tabular}

Table 21: Comparative studies in acute coronary syndromes, safety results, bleeding. 


\section{Acknowledgement}

We are grateful for the useful comments and information following our request for a check on scientific correctness and completeness of the manuscript from the manufacturers of the DOACs: Bayer, BMS/Pfizer, Boehringer Ingelheim and Daiichi Sankyo. The score is based on the opinions of the authors and does not necessarily reflect the opinions of these companies.

\section{Conflicts of Interest}

None reported.

\section{Bibliography}

1. Janknegt R and Steenhoek A. "The system of Objectified Judgement Analysis. A tool in rational drug selection for formulary inclusion". Drugs 53 (1997): 551-562.

2. Summary of Product Characteristics. Apixaban (Eliquis).

3. Summary of Product Characteristics. Dabigratran (Pradaxa).

4. Summary of Product Characteristics. Edoxaban (Lixiana).

5. Summary of Product Characteristics. Rivaroxaban (Xarelto).

6. Frost C., et al. "A randomized direct comparison of the pharmacokinetics and pharmacodynamics of apixaban and rivaroxaban". Clinical Pharmacology 6 (2014): 179-187.

7. Frost C., et al. "Evaluation of the effect of naproxen on the pharmacokinetics and pharmacodynamics of apixaban". British Journal of Clinical Pharmacology 78 (2014): 877-885.

8. Wang X., et al. "Effect of activated charcoal on apixaban pharmacokinetics in healthy subjects". American Journal of Cardiovascular Drugs 4.14 (2014): 147-154.

9. Upreti VV., et al. "Effect of famotidine on the pharmacokinetics of apixaban, an oral direct factor Xa inhibitor". Clinical Pharmacology 5 (2013): 59-66.

10. Clemens A., et al. "Switching from enoxaparin to dabigatran etexilate: pharmacokinetics, pharmacodynamics, and safety profile". European Journal of Clinical Pharmacology 68 (2012): 607-616.

11. Stangier J., et al. "Pharmacokinetics and pharmacodynamics of dabigatran etexilate, an oral direct thrombin inhibitor, with coadministration of digoxin". The Journal of Clinical Pharmacology 52 (2012): 243-250.
12. Stangier J., et al. "Pharmacokinetics and pharmacodynamics of dabigatran etexilate, an oral direct thrombin inhibitor, are not affected by moderate hepatic impairment". The Journal of Clinical Pharmacology 48 (2008): 1411-1419.

13. Wilson JA., et al. "An evaluation of oral dabigatran etexilate pharmacokinetics and pharmacodynamics in hemodialysis". The Journal of Clinical Pharmacology 54 (2014): 901-909.

14. Parasrampuria DA., et al. "Pharmacokinetics and Pharmacodynamics of the Nonvitamin K Antagonist Oral Anticoagulant Edoxaban When Administered Alone or After Switching from Rivaroxaban or Dabigatran Etexilate in Healthy Subjects". Clinical Drug Investigation 36 (2016): 127-136.

15. Mendell J., et al. "Drug-drug interaction studies of cardiovascular drugs involving P-glycoprotein, an efflux transporter, on the pharmacokinetics of edoxaban, an oral factor Xa inhibitor". American Journal of Cardiovascular Drugs 13 (2013): 331-342.

16. Bathala MS., et al. "Pharmacokinetics, biotransformation, and mass balance of edoxaban, a selective, direct factor Xa inhibitor, in humans". Drug Metabolism and Disposition 40 (2012): 2250-2255.

17. Mendell J., et al. "A randomized trial of the safety, pharmacokinetics and pharmacodynamics of edoxaban, an oral factor Xa inhibitor, following a switch from warfarin". British Journal of Clinical Pharmacology 75 (2013): 966-978.

18. Moore KT., et al. "An open-label study to estimate the effect of steady-state erythromycin on the pharmacokinetics, pharmacodynamics, and safety of a single dose of rivaroxaban in subjects with renal impairment and normal renal function". The Journal of Clinical Pharmacology 54 (2014): 1407-1420.

19. Kubitza D., et al. "Effect of hepatic impairment on the pharmacokinetics and pharmacodynamics of a single dose of rivaroxaban, an oral, direct Factor Xa inhibitor". British Journal of Clinical Pharmacology 76 (2013): 89-98.

20. Zhao X., et al. "Safety, pharmacokinetics and pharmacodynamics of single/multiple doses of the oral, direct Factor Xa inhibitor rivaroxaban in healthy Chinese subjects". British Journal of Clinical Pharmacology 68 (2009): 77-88.

21. Kreutz R., et al. "Dissociation between the pharmacokinetics and pharmacodynamics of once-daily rivaroxaban and twicedaily apixaban: a randomized crossover study". Journal of Thrombosis and Haemostasis 15 (2017): 2017-2028. 
22. Frost CE., et al. "Effect of ketoconazole and diltiazem on the pharmacokinetics of apixaban, an oral direct factor Xa inhibitor". British Journal of Clinical Pharmacology 79 (2015): 838846.

23. Mendell J., et al. "The effect of rifampin on the pharmacokinetics of edoxaban in healthy adults". Clinical Drug Investigation 35 (2015): 447-453.

24. Vakkalagadda B., et al. "Effect of Rifampin on the Pharmacokinetics of Apixaban, an Oral Direct Inhibitor of Factor Xa". American Journal of Cardiovascular Drugs (2016).

25. Chang SH., et al. "Association Between Use of Non-Vitamin $\mathrm{K}$ Oral Anticoagulants With and Without Concurrent Medications and Risk of Major Bleeding in Nonvalvular Atrial Fibrillation". JAMA 318 (2017): 1250-1259.

26. Lassen MR., et al. "The efficacy and safety of apixaban, an oral, direct factor Xa inhibitor, as thromboprophylaxis in patients following total knee replacement". Journal of Thrombosis and Haemostasis 5 (2007): 2368-2375.

27. Lassen MR., et al. "Apixaban or enoxaparin for thromboprophylaxis after knee replacement". The New England Journal of Medicine 361 (2009): 594-604.

28. Lassen MR., et al. "Apixaban versus enoxaparin for thromboprophylaxis after knee replacement (ADVANCE-2): a randomised double-blind trial". Lancet 37 (2010): 807-815.

29. Lassen MR., et al. "Apixaban versus enoxaparin for thromboprophylaxis after hip replacement". The New England Journal of Medicine 363 (2010): 2487-2498.

30. Eriksson BI., et al. "A new oral direct thrombin inhibitor, dabigatran etexilate, compared with enoxaparin for prevention of thromboembolic events following total hip or knee replacement: the BISTRO II randomized trial". Journal of Thrombosis and Haemostasis 3 (2005): 103-111.

31. Eriksson BI., et al. "Oral dabigatran etexilate vs. subcutaneous enoxaparin for the prevention of venous thromboembolism after total knee replacement: the RE-MODEL randomized trial". Journal of Thrombosis and Haemostasis 5 (2007): 2178-2185.

32. Eriksson BI., et al. "Dabigatran etexilate versus enoxaparin for prevention of venous thromboembolism after total hip replacement: a randomised, double-blind, non-inferiority trial". Lancet 370 (2007): 949-956.
33. Eriksson BI., et al. "Oral dabigatran versus enoxaparin for thromboprophylaxis after primary total hip arthroplasty (RENOVATE II*). A randomised, double-blind, non-inferiority trial". Thrombosis and Haemostasis 105 (2011): 721-729.

34. RE-MOBILIZE Writing Committee. "Oral thrombin inhibitor dabigatran etexilate vs North American enoxaparin regimen for prevention of venous thromboembolism after knee arthroplasty surgery". Journal of Arthroplasty 24 (2009): 1-9.

35. Raskob G., et al. "Oral direct factor Xa inhibition with edoxaban for thromboprophylaxis after elective total hip replacement. A randomised double-blind dose-response study". Thrombosis and Haemostasis 104 (2010): 642-649.

36. Fuji T., et al. "Safety and efficacy of edoxaban, an oral factor Xa inhibitor, versus enoxaparin for thromboprophylaxis after total knee arthroplasty: the STARS E-3 trial". Thrombosis Research 134 (2014): 1198-1204.

37. Fuji T., et al. "Safety and efficacy of edoxaban, an oral factor xa inhibitor, for thromboprophylaxis after total hip arthroplasty in Japan and Taiwan". Journal of Arthroplasty 29 (2014): 24392446.

38. Fuji T., et al. "Efficacy and safety of edoxaban versus enoxaparin for the prevention of venous thromboembolism following total hip arthroplasty: STARS J-V". Thrombosis Journal 13 (2015): 27.

39. Eriksson BI., et al. "Rivaroxaban versus enoxaparin for thromboprophylaxis after hip arthroplasty". The New England Journal of Medicine 358 (2008): 2765-2775.

40. Kakkar AK., et al. "Extended duration rivaroxaban versus short-term enoxaparin for the prevention of venous thromboembolism after total hip arthroplasty: a double-blind, randomized controlled trial". Lancet 372 (2008): 31-39.

41. Lassen MR., et al. "Rivaroxaban versus enoxaparin for thromboprophylaxis after total knee arthroplasty". The New England Journal of Medicine 358 (2008): 2776-2786.

42. Turpie AG., et al. "Rivaroxaban versus enoxaparin for thromboprophylaxis after total knee arthroplasty (RECORD4): a randomised trial". Lancet 373 (2009): 1673-1680.

43. Anderson DR., et al. "Aspirin or Rivaroxaban for VTE Prophylaxis after Hip or Knee Arthroplasty". The New England Journal of Medicine 378 (2018): 699-707. 
44. Connolly SJ., et al. "Apixaban in patients with atrial fibrillation". The New England Journal of Medicine 364 (2011): 806-817.

45. Granger CB., et al. "Apixaban versus warfarin in patients with atrial fibrillation". The New England Journal of Medicine 365 (2011): 981-992.

46. Connolly SJ., et al. "Dabigatran versus warfarin in patients with atrial fibrillation". The New England Journal of Medicine 361 (2009): 1139-1151.

47. Giugliano RP., et al. "Edoxaban versus warfarin in patients with atrial fibrillation". The New England Journal of Medicine 369 (2013): 2093-2104.

48. Calkins H., et al. "Uninterrupted dabigatran versus warfarin for ablation in atrial fibrillation". The New England Journal of Medicine 376 (2017): 1627-1636.

49. Cannon CP., et al. "Dual antithrombotic therapy with dabigatran after PCI in atrial fibrillation". The New England Journal of Medicine 37 (2017): 1513-1524.

50. Patel MR., et al. "Rivaroxaban versus warfarin in nonvalvular atrial fibrillation". The New England Journal of Medicine 365 (2011): 883-891.

51. Cappato R., et al. "Rivaroxaban vs. vitamin $\mathrm{K}$ antagonists for cardioversion in atrial fibrillation". European Heart Journal 35 (2014): 3346-3355.

52. Agnelli G., et al. "Oral apixaban for the treatment of acute venous thromboembolism". The New England Journal of Medicine 369 (2013): 799-808.

53. Agnelli G., et al. "Apixaban for extended treatment of venous thromboembolism". The New England Journal of Medicine 368 (2013): 699-708.

54. Goldhaber SZ., et al. "Apixaban versus enoxaparin for thromboprophylaxis in medically ill patients". The New England Journal of Medicine 365 (2011): 2167-2177.

55. Schulman S., et al. "Dabigatran versus warfarin in the treatment of acute venous thromboembolism". The New England Journal of Medicine 361 (2009): 2342-2352.

56. Schulman S., et al. "Treatment of acute venous thromboembolism with dabigatran or warfarin and pooled analysis". Circulation 129 (2014): 764-772.

57. Schulman S., et al. "Extended use of dabigatran, warfarin, or placebo in venous thromboembolism". The New England Journal of Medicine 368 (2013): 709-718.
58. Hokusai-VTE Investigators., et al. "Edoxaban versus warfarin for the treatment of symptomatic venous thromboembolism". The New England Journal of Medicine 369 (2013): 1406-1415.

59. Raskob GE., et al. "Edoxaban for the Treatment of Cancer-Associated Venous Thromboembolism". The New England Journal of Medicine 378 (2018): 615-624.

60. EINSTEIN Investigators., et al. "Oral rivaroxaban for symptomatic venous thromboembolism". The New England Journal of Medicine 363 (2010): 2499-2510.

61. EINSTEIN-PE Investigators., et al. "Oral rivaroxaban for the treatment of symptomatic pulmonary embolism". The New England Journal of Medicine 366 (2012): 1287-1297.

62. Cohen AT., et al. "Rivaroxaban for thromboprophylaxis in acutely ill medical patients". The New England Journal of Medicine 368 (2013): 513-523.

63. Weitz JI., et al. "Rivaroxaban or aspirin for extended treatment of venous thromboembolism". The New England Journal of Medicine 376 (2017): 1211-22.

64. Alexander JH., et al. "Apixaban with antiplatelet therapy after acute coronary syndrome". The New England Journal of Medicine 365 (2011): 699-708.

65. Mega JL., et al. "Rivaroxaban in patients with a recent acute coronary syndrome". The New England Journal of Medicine 366 (2012): 9-19.

66. Ohman EM., et al. "Clinically significant bleeding with lowdose rivaroxaban versus aspirin, in addition to P2Y12 inhibition, in acute coronary syndromes (GEMINI-ACS-1): a double-blind, multicentre, randomised trial". Lancet 389 (2017): 1799-1808.

67. Eriksson BI., et al. "Dose escalating safety study of a new oral direct thrombin inhibitor, dabigatran etexilate, in patients undergoing total hip replacement: BISTRO I". Journal of Thrombosis and Haemostasis 2 (2004): 1573-1580.

68. Fuji T., et al. "Dabigatran etexilate prevents venous thromboembolism after total knee arthroplasty in Japanese patients with a safety profile comparable to placebo". Journal of Arthroplasty 25 (2010): 1267-1274.

69. Eriksson BI., et al. "Oral dabigatran etexilate versus enoxaparin for venous thromboembolism prevention after total hip arthroplasty: pooled analysis of two phase 3 randomized trials". Thrombosis Journal 13 (2015): 36. 
70. Friedman RJ., et al. "Dabigatran versus enoxaparin for prevention of venous thromboembolism after hip or knee arthroplasty: a pooled analysis of three trials". Thrombosis Research 126 (2010): 175-182.

71. Wolowacz SE., et al. "Efficacy and safety of dabigatran etexilate for the prevention of venous thromboembolism following total hip or knee arthroplasty. A meta-analysis". Thrombosis and Haemostasis 101 (2009): 77-85.

72. Fuji T., et al. "A dose-ranging study evaluating the oral factor Xa inhibitor edoxaban for the prevention of venous thromboembolism in patients undergoing total knee arthroplasty". Journal of Thrombosis and Haemostasis 8 (2010): 2458-2468.

73. Fuji T., et al. "Safety and efficacy of edoxaban in patients undergoing hip fracture surgery". Thrombosis Research 133 (2014): 1016-1022.

74. Seife C. "Research misconduct identified by the US Food and Drug Administration: out of sight, out of mind, out of the peerreviewed literature". JAMA Internal Medicine 175 (2015): 567577.

75. Huang HF., et al. "Rivaroxaban versus enoxaparin for the prevention of venous thromboembolism after total knee arthroplasty: A meta-analysis". Medicine (Baltimore) 97 (2018): e13465.

76. López-López JA., et al. "Oral anticoagulants for prevention of stroke in atrial fibrillation: systematic review, network metaanalysis, and cost effectiveness analysis". British Medical Journal 359 (2017): j5058.

77. O’Donnell MJ., et al. "Effect of apixaban on brain infarction and microbleeds: AVERROES-MRI assessment study". American Heart Journal 178 (2016): 145-150.

78. Easton JD., et al. "Apixaban compared with warfarin in patients with atrial fibrillation and previous stroke or transient ischaemic attack: a subgroup analysis of the ARISTOTLE trial". Lancet Neurology 11 (2012): 503-511.

79. Avezum A., et al. "Apixaban in Comparison With Warfarin in Patients With Atrial Fibrillation and Valvular Heart Disease: Findings From the Apixaban for Reduction in Stroke and Other Thromboembolic Events in Atrial Fibrillation (ARISTOTLE) Trial". Circulation 132 (2015): 624-632.

80. Garmendia CA., et al. "Evaluation of the Inclusion of Studies Identified by the FDA as Having Falsified Data in the Results of Meta-analyses: The Example of the Apixaban Trials". JAMA Internal Medicine 179 (2019): 582-584.
81. Ezekowitz MD., et al. "Comparison of Dabigatran and Warfarin in Patients With Atrial Fibrillation and Valvular Heart Disease: The RE-LY Trial (Randomized Evaluation of Long-Term Anticoagulant Therapy)". Circulation 134 (2016): 589-598.

82. Connolly SJ., et al. "The Long-Term Multicenter Observational Study of Dabigatran Treatment in Patients With Atrial Fibrillation (RELY-ABLE) Study". Circulation 128 (2013): 237-243.

83. Lip G., et al. "Patient outcomes using the European label for dabigatran. A post-hoc analysis from the RE-LY database". Thrombosis and Haemostasis 111 (2014): 933-942.

84. Brambatti M., et al. "Comparison of dabigatran versus warfarin in diabetic patients with atrial fibrillation: Results from the RE-LY trial". International Journal of Cardiology 196 (2015): 127-131.

85. Seeger JD., et al. "Safety and effectiveness of dabigatran and warfarin in routine care of patients with atrial fibrillation". Thrombosis and Haemostasis 114 (2015): 1277-1289.

86. Larsen TB., et al. "Dabigatran and warfarin for secondary prevention of stroke in atrial fibrillation patients: a nationwide cohort study". American Journal of Medicine 127 (2014): 11721178.

87. Del-Carpio Munoz F., et al. "Dabigatran Versus Warfarin in Relation to Renal Function in Patients With Atrial Fibrillation". Journal of the American College of Cardiology 68 (2016): 129131.

88. Giugliano RP., et al. "Mortality in Patients with Atrial Fibrillation Randomized to Edoxaban or Warfarin: Insights from the ENGAGE AF-TIMI 48 Trial". American Journal of Medicine 129 (2016): 850-857.

89. Giugliano RP., et al. “Cerebrovascular events in 21105 patients with atrial fibrillation randomized to edoxaban versus warfarin: Effective Anticoagulation with Factor Xa Next Generation in Atrial Fibrillation-Thrombolysis in Myocardial Infarction 48". Stroke 45 (2014): 2372-2378.

90. Geller BJ., et al. "Systemic, noncerebral, arterial embolism in 21,105 patients with atrial fibrillation randomized to edoxaban or warfarin: results from the Effective Anticoagulation With Factor Xa Next Generation in Atrial Fibrillation-Thrombolysis in Myocardial Infarction Study 48 trial". American heart Journal 170 (2015): 669-674.

91. Giugliano RP., et al. "Mortality in Patients With Atrial Fibrillation Randomized to Edoxaban or Warfarin: Insights from the ENGAGE AF-TIMI 48 Trial". American Journal of Medicine (2016): pii: S0002-9343 (16)30246-7. 
92. Steffel J., et al. "Edoxaban Versus Warfarin in Atrial Fibrillation Patients at Risk of Falling: ENGAGE AF-TIMI 48 Analysis". Journal of the American College of Cardiology 68 (2016): 11691178.

93. Rost NS., et al. "Outcomes With Edoxaban Versus Warfarin in Patients With Previous Cerebrovascular Events: Findings From ENGAGE AF-TIMI 48 (Effective Anticoagulation With Factor Xa Next Generation in Atrial Fibrillation-Thrombolysis in Myocardial Infarction 48)". Stroke 47 (2016): 2075-2082.

94. Weitz JI., et al. "Randomised, parallel-group, multicentre, multinational phase 2 study comparing edoxaban, an oral factor Xa inhibitor, with warfarin for stroke prevention in patients with atrial fibrillation". Thrombosis and Haemostasis 104 (2010): 633-641.

95. Goette A., et al. "Edoxaban versus enoxaparin-warfarin in patients undergoing cardioversion of atrial fibrillation (ENSUREAF): a randomised, open-label, phase 3b trial". Lancet 388 (2016): 1995-2003.

96. Hankey GJ., et al. "Rivaroxaban compared with warfarin in patients with atrial fibrillation and previous stroke or transient ischaemic attack: a subgroup analysis of ROCKET AF". Lancet Neurology 11 (2012): 315-322.

97. Halperin JL., et al. "Efficacy and safety of rivaroxaban compared with warfarin among elderly patients with nonvalvular atrial fibrillation in the Rivaroxaban Once Daily, Oral, Direct Factor Xa Inhibition Compared With Vitamin K Antagonism for Prevention of Stroke and Embolism Trial in Atrial Fibrillation (ROCKET AF)". Circulation 130 (2014): 138-146.

98. Piccini JP., et al. "Relationship between time in therapeutic range and comparative treatment effect of rivaroxaban and warfarin: results from the ROCKET AF trial". Journal of the American Heart Association 3 (2014): e000521.

99. Bansilal S., et al. "Efficacy and safety of rivaroxaban in patients with diabetes and nonvalvular atrial fibrillation: the Rivaroxaban Once-daily, Oral, Direct Factor Xa Inhibition Compared with Vitamin K Antagonism for Prevention of Stroke and Embolism Trial in Atrial Fibrillation (ROCKET AF Trial)". American Heart Journal 170 (2015): 675-682.

100. Fordyce CB., et al. "On-Treatment Outcomes in Patients With Worsening Renal Function With Rivaroxaban Compared With Warfarin: Insights From ROCKET AF". Circulation 134 (2016): $37-47$.
101. Lin LL., et al. "Clinical and safety outcomes of oral antithrombotics for stroke prevention in atrial fibrillation: a systematic review and network meta-analysis". Journal of the American Medical Directors Association 16 (2015): 1103.e1-19.

102. Graham DJ., et al. "Stroke, Bleeding, and Mortality Risks in Elderly Medicare Beneficiaries Treated With Dabigatran or Rivaroxaban for Nonvalvular Atrial Fibrillation". JAMA Internal Medicine 176 (2016): 1662-1671.

103. Healthcare Improvement Scotland. "A review of the clinical effectiveness of direct oral anticoagulants for the prevention of stroke and systemic embolism in adult patients with nonvalvular atrial fibrillation". www.healthcareimprovementscotland.org (2017).

104. Chan YH., et al. "Thromboembolic, Bleeding, and Mortality Risks of Rivaroxaban and Dabigatran in Asians With Nonvalvular Atrial Fibrillation". Journal of the American College of Cardiology 68 (2016): 1389-1401.

105. Jacobs V., et al. "Long-Term Population-Based Cerebral Ischemic Event and Cognitive Outcomes of Direct Oral Anticoagulants Compared With Warfarin Among Long-term Anticoagulated Patients for Atrial Fibrillation". American Journal of Cardiology 118 (2016): 210-214.

106. Larsen TB., et al. "Comparative effectiveness and safety of non-vitamin $\mathrm{K}$ antagonist oral anticoagulants and warfarin in patients with atrial fibrillation: propensity weighted nationwidecohort study". British Medical Journal 353 (2016): i3189.

107. Larsen TB., et al. "Effectiveness and safety of reduced dose non-vitamin $\mathrm{K}$ antagonist oral anticoagulants and warfarin in patients with atrial fibrillation: propensity weighted nationwide cohort study". British Medical Journal 356 (2017): j510.

108. Coleman CI., et al. "Real-world evidence of stroke prevention in patients with nonvalvular atrial fibrillation in the United States: the Revisit-US study". Current Medical Research and Opinion (2016).

109. Hernandez I., et al. "Comparison of the effectiveness and safety of apixaban, dabigatran, rivaroxaban and warfarin in newly diagnosed atrial fibrillation". American Journal of Cardiology 120 (2017): 1813-1819.

110. Lip G., et al. "Effectiveness and Safety of Oral Anticoagulants Among Nonvalvular Atrial Fibrillation Patients. The ARISTOPHANES Study". Stroke 49 (2018): 2933-2944. 
111. Graham DJ., et al. “Comparative Stroke, Bleeding, and Mortality Risks in Older Medicare Patients Treated with Oral Anticoagulants for Nonvalvular Atrial Fibrillation". American Journal of Medicine 132 (2019): 596-604.

112. Liu X., et al. "Apixaban Reduces Hospitalizations in Patients With Venous Thromboembolism: An Analysis of the Apixaban for the Initial Management of Pulmonary Embolism and DeepVein Thrombosis as First-Line Therapy (AMPLIFY) Trial". Journal of the American Heart Association 4 (2015): pii: e002340.

113. Agnelli G., et al. "Oral apixaban for the treatment of venous thromboembolism in cancer patients: results from the AMPLIFY trial". Journal of Thrombosis and Haemostasis 13 (2015): 2187-2191.

114. Botticelli Investigators., et al. "Efficacy and safety of the oral direct factor Xa inhibitor apixaban for symptomatic deep vein thrombosis. The Botticelli DVT dose-ranging study". Journal of Thrombosis and Haemostasis 6 (2008): 1313-1318.

115. Agnelli G., et al. "Treatment of proximal deep-vein thrombosis with the oral direct factor Xa inhibitor rivaroxaban (BAY 597939): the ODIXa-DVT (Oral Direct Factor Xa Inhibitor BAY 597939 in Patients With Acute Symptomatic Deep-Vein Thrombosis) study". Circulation 116 (2007): 180-187.

116. Buller HR., et al. "A dose-ranging study evaluating once-daily oral administration of the factor Xa inhibitor rivaroxaban in the treatment of patients with acute symptomatic deep vein thrombosis: the Einstein-DVT Dose-Ranging Study". Blood 112 (2008): 2242-2247.

117. Robertson R., et al. "Oral direct thrombin inhibitors or oral factor Xa inhibitors for the treatment of pulmonary embolism". Cochrane Database of Systematic Reviews 12 (2015).

118. Robertson R., et al. "Oral direct thrombin inhibitors or oral factor Xa inhibitors for the treatment of deep venous thrombosis". Cochrane Database of Systematic Reviews 6 (2015): CD010956.

119. APPRAISE Steering Committee and Investigators., et al. "Apixaban, an oral, direct, selective factor Xa inhibitor, in combination with antiplatelet therapy after acute coronary syndrome: results of the Apixaban for Prevention of Acute Ischemic and Safety Events (APPRAISE) trial". Circulation 119 (2009): 28772885.

120. Oldren J., et al. "Dabigatran vs placebo in patients with acute coronary syndromes on dual antiplatelet therapy: a randomized, double-blind, phase II trial". European Heart Journal 32 (2011): 2781-2789.
121. Mega JL., et al. "Rivaroxaban versus placebo in patients with acute coronary syndromes (ATLAS ACS-TIMI 46): a randomised, double-blind, phase II trial". Lancet 374 (2009): 2938.

122. Eikelboom JW., et al. "Rivaroxaban with or without Aspirin in Stable Cardiovascular Disease". The New England Journal of Medicine 377 (2017): 1319-1330.

123. Connolly SJ., et al. "Rivaroxaban with or without aspirin in patients with stable coronary artery disease: an international, randomised, double-blind, placebo-controlled trial". Lancet 391 (2018): 205-218.

124. Anand SS., et al. "Rivaroxaban with or without aspirin in patients with stable peripheral or carotid artery disease: an international, randomised, double-blind, placebo-controlled trial”. Lancet 391 (2018): 219-229.

125. López-López JA., et al. “Oral anticoagulants for prevention of stroke in atrial fibrillation: systematic review, network metaanalysis, and cost effectiveness analysis". British Medical Journal 359 (2017): j5058.

126. Hylek EM., et al. "Major bleeding in patients with atrial fibrillation receiving apixaban or warfarin: The ARISTOTLE Trial (Apixaban for Reduction in Stroke and Other Thromboembolic Events in Atrial Fibrillation): Predictors, Characteristics, and Clinical Outcomes". Journal of the American College of Cardiology 63 (2014): 2141-2147.

127. De Caterina R., et al. "History of bleeding and outcomes with apixaban versus warfarin in patients with atrial fibrillation in the Apixaban for Reduction in Stroke and Other Thromboembolic Events in Atrial Fibrillation trial". American Heart Journal 175 (2016): 175-183.

128. Villa LA., et al. "Evaluating the efficacy and safety of apixaban, a new oral anticoagulant, using Bayesian meta-analysis". International Journal of Hematology 98 (2013): 390-397.

129. Touma L., et al. "A meta-analysis of randomized controlled trials of the risk of bleeding with apixaban versus vitamin $\mathrm{K}$ antagonists". American Journal of Cardiology 115 (2015): 533541.

130. Mantha S and Ansell J. "Indirect comparison of dabigatran, rivaroxaban, apixaban and edoxaban for the treatment of acute venous thromboembolism". Journal of Thrombosis and Thrombolysis 39 (2015): 155-165. 
131. Cohen AT., et al. "Comparison of the Novel Oral Anticoagulants Apixaban, Dabigatran, Edoxaban, and Rivaroxaban in the Initial and Long-Term Treatment and Prevention of Venous Thromboembolism: Systematic Review and Network MetaAnalysis". PLoS One 10 (2015): e0144856.

132. Kolb JM., et al. "Locations and Mucosal Lesions Responsible for Major Gastrointestinal Bleeding in Patients on Warfarin or Dabigatran". Digestive Diseases and Science 63 (2018): 18781889.

133. Majeed A., et al. "Bleeding events with dabigatran or warfarin in patients with venous thromboembolism". Thrombosis and Haemostasis 115 (2015): 291-298.

134. Nishtala PS., et al. "'Real-world' haemorrhagic rates for warfarin and dabigatran using population-level data in New Zealand". International Journal of Cardiology 203 (2015): 746-752.

135. Maura G., et al. "Comparison of the Short-Term Risk of Bleeding and Arterial Thromboembolic Events in Nonvalvular Atrial Fibrillation Patients Newly Treated With Dabigatran or Rivaroxaban Versus Vitamin K Antagonists: A French Nationwide Propensity-Matched Cohort Study". Circulation 132 (2015): 1252-1260.

136. Fontaine GV., et al. "Major bleeding with dabigatran and rivaroxaban in patients with atrial fibrillation: a real-world setting". Clinical and Applied Thrombosis/Hemostasis 20 (2014): 665672.

137. Abraham NS., et al. "Comparative risk of gastrointestinal bleeding with dabigatran, rivaroxaban, and warfarin: population based cohort study". British Medical Journal 350 (2015): h1857.

138. Douxfils J., et al. "Dabigatran etexilate and risk of myocardial infarction, other cardiovascular events, major bleeding, and all-cause mortality: a systematic review and meta-analysis of randomized controlled trials". Journal of the American Heart Association 3 (2014): e000515.

139. Wang SV., et al. "Prediction of rates of thromboembolic and major bleeding outcomes with dabigatran or warfarin among patients with atrial fibrillation: new initiator cohort study". British Medical Journal 353 (2016): i2607.

140. Chung N., et al. "Safety of edoxaban, an oral factor Xa inhibitor, in Asian patients with non-valvular atrial fibrillation". Thrombosis and Haemostasis 105 (2011): 535-544.

141. Li S., et al. "Bleeding risk and mortality of edoxaban: a pooled meta-analysis of randomized controlled trials". PLoS One 9 (2014): e95354.
142. Sherwood MW., et al. "Gastrointestinal Bleeding in Patients With Atrial Fibrillation Treated With Rivaroxaban or Warfarin: ROCKET AF Trial". Journal of the American College of Cardiology 66 (2015): 2271-2281.

143. Lip GY., et al. "Real-world comparison of major bleeding risk among non-valvular atrial fibrillation patients initiated on apixaban, dabigatran, rivaroxaban, or warfarin. A propensity score matched analysis". Thrombosis and Haemostasis 116 (2016): 975-986.

144. Maura G., et al. "Comparison of the short-term risk of bleeding and arterial thromboembolic events in nonvalvular atrial fibrillation patients newly treated with dabigatran or rivaroxaban versus vitamin $\mathrm{K}$ antagonists: a French nationwide propensity-matched cohort study". Circulation 132 (2015): 1252-1260.

145. Lip GY., et al. "Major bleeding risk among non-valvular atrial fibrillation patients initiated on apixaban, dabigatran, rivaroxaban or warfarin: a "real-world" observational study in the United States". International Journal of Clinical Practice 70 (2016): 752-763.

146. Bundhun PK., et al. "Bleeding outcomes associated with rivaroxaban and dabigatran in patients treated for atrial fibrillation: a systematic review and meta-analysis". BMC Cardiovascular Disorder 17 (2017): 15.

147. Lai CL., et al. "Comparative Effectiveness and Safety of Dabigatran and Rivaroxaban in Atrial Fibrillation Patients". Journal of the American Heart Association 6.4 (2017).

148. Gorst-Rasmussen A., et al. "Rivaroxaban versus warfarin and dabigatran in atrial fibrillation: comparative effectiveness and safety in Danish routine care". Pharmacoepidemiology and Drug Safety 25 (2016): 1236-1244.

149. Noseworthy PA., et al. "Direct Comparison of Dabigatran, Rivaroxaban, and Apixaban for Effectiveness and Safety in Nonvalvular Atrial Fibrillation". Chest 150 (2016): 1302-1312.

150. Yao X., et al. "Effectiveness and Safety of Dabigatran, Rivaroxaban, and Apixaban Versus Warfarin in Nonvalvular Atrial Fibrillation". Journal of the American Heart Association 5.6 (2016).

151. Li WH., et al. "Efficacy and safety of dabigatran, rivaroxaban, and warfarin for stroke prevention in Chinese patients with atrial fibrillation: the Hong Kong Atrial Fibrillation Project". Clinical Cardiology 40 (2017): 222-229. 
152. Lip GY., et al. "Relative efficacy and safety of non-Vitamin K oral anticoagulants for non-valvular atrial fibrillation: Network meta-analysis comparing apixaban, dabigatran, rivaroxaban and edoxaban in three patient subgroups". International Journal of Cardiology 204 (2016): 88-94.

153. Abraham NS., et al. "Gastrointestinal Safety of Direct Oral Anticoagulants: A Large Population-Based Study". Gastroenterology 152 (2017): 1014-1022.e1.

154. Di Nisio M., et al. "Risk of major bleeding in patients with venous thromboembolism treated with rivaroxaban or with heparin and vitamin K antagonists". Thrombosis and Haemostasis 115.2 (2016): 424-432.

155. Eerenberg ES., et al. "Clinical impact and course of major bleeding with rivaroxaban and vitamin K antagonists". Journal of Thrombosis and Haemostasis 13 (2015): 1590-1596.

156. Husted S., et al. "Reversal Strategies for NOACs: State of Development, Possible Clinical Applications and Future Perspectives". Drug Safety 39 (2016): 5-13.

157. Connolly SJ., et al. "Andexanet Alfa for Acute Major Bleeding Associated with Factor Xa Inhibitors". The New England Journal of Medicine 375 (2016): 1131-1141.

158. Siegal DM., et al. "Adnexanet alfa for the reversal of Factor Xa inhibitor activity". The New England Journal of Medicine 373 (2015): 2413-2424.

159. Beyer-Westendorf J., et al. "Rates, management, and outcome of rivaroxaban bleeding in daily care: results from the Dresden NOAC registry". Blood 124 (2014): 955-962.

160. Hohnloser SH., et al. "Myocardial ischemic events in patients with atrial fibrillation treated with dabigatran or warfarin in the RE-LY (Randomized Evaluation of Long-Term Anticoagulation Therapy) trial". Circulation 125 (2012): 669-676.

161. Uchino K., et al. "Dabigatran association with higher risk of acute coronary events: meta-analysis of noninferiority randomized controlled trials". Archives of Internal Medicine 172 (2012): 397-402.

162. Wei AH., et al. "Increased risk of myocardial infarction with dabigatran etexilate: fact or fiction? A critical meta-analysis of over 580,000 patients from integrating randomizedcontrolled trials and real-world studies". International Journal of Cardiology 267 (2018): 1-7.

163. Villines TC., et al. "A comparison of the safety and effectiveness of dabigatran and warfarin in non-valvular atrial fibrillation patients in a large healthcare system". Thrombosis and Haemostasis 114 (2015): 1290-1298.
164. Lambert A., et al. "Rivaroxaban-induced liver injury: Results from a venous thromboembolism registry". International Journal of Cardiology 191 (2015): 265-266.

165. Liakoni E., et al. "Hepatotoxicity of New Oral Anticoagulants (NOACs)". Drug Safety 38 (2015): 711-720.

166. Raparelli V., et al. "Adherence to oral anticoagulant therapy in patients with atrial fibrillation". Thrombosis and Haemostasis 117.2 (2017): 209-218.

167. Coleman GI., et al. "Medication adherence to rivaroxaban and dabigatran for stroke prevention in patients with non-valvular atrial fibrillation in the United States". International Journal of Cardiology 212 (2016): 171-173.

168. Mc Horney CA., et al. "Comparison of adherence to rivaroxaban versus apixaban among patients with atrial fibrillation". Clinical Therapy 38 (2016): 2477-2488.

169. Sorensen R., et al. "Adherence with oral anticoagulation in non-valvular atrial fibrillation: a comparison of vitamin $\mathrm{K}$ antagonists and non-vitamine K antagonists". European Heart Journal 3.3 (2017): 151-156.

\section{Assets from publication with us}

- Prompt Acknowledgement after receiving the article

- Thorough Double blinded peer review

- Rapid Publication

- Issue of Publication Certificate

- High visibility of your Published work

Website: www.actascientific.com/

Submit Article: www.actascientific.com/submission.php

Email us: editor@actascientific.com

Contact us: +919182824667 